\title{
Woman-Centered Design through Humanity, Activism, and Inclusion
}

\author{
Teresa Almeida \\ IxD Lab, IT University of Copenhagen, Copenhagen, Denmark, teal@itu.dk \\ Madeline Balaam \\ MID, KTH Royal Institute of Technology, Stockholm, Sweden, balaam@kth.se \\ Rob Comber \\ MID, KTH Royal Institute of Technology, Stockholm, Sweden, rcomber@kth.se
}

\begin{abstract}
Women account for over half of the global population, however continue to be subject to systematic and systemic disadvantage, particularly in terms of access to health and education. At every intersection, where systemic inequality accounts for greater loss of life or limitations on full and healthy living, women are more greatly impacted by those inequalities. The design of technologies is no different, the very definition of technology is historically cast in terms of male activities, and advancements in the field are critical to improve women's quality of life. This paper views HCI, a relatively new field, as well positioned to act critically in the ways that technology serve, refigure and redefine women's bodies. Indeed, the female body remains a contested topic, a restriction to the development of women's health. Women's health - as a medicalized field exists, and people's experiences of it are problematic for many. This is visible today in e.g. socio-cultural practices in disparate geographies or medical devices within a clinic. Moreover, the biological body is part of a great unmentionable, i.e. the perils of essentialism. We contend that it is necessary, pragmatically and ethically, for HCI to turn its attention towards a woman-centered design approach. While previous research has argued for the dangers of gender-demarcated design work, we advance that designing for and with women should not be regarded as ghettoizing, but instead as critical to improving women's experiences in bodily transactions, choices, rights, and access to and in health and care. In this paper, we consider how and why designing with and for Woman matters, and use our design-led research as a way to speak to, and illustrate alternatives to this field.
\end{abstract}

CCS CONCEPT insertion: The new template enables you to import required indexing concepts for your article from the ACM Computing Classification System (CCS) using an indexing support tool found in the ACM Digital Library (DL). The tool generates formatted text after you have selected your terms. To insert your CCS terms into your document, copy and paste the formatted text from the CCS tool using the "https://dl.acm.org/ccs/ccs.cfm" link into the "CCS CONCEPTS" section.

\section{CCS CONCEPTS}

- Insert your first CCS term here $\bullet$ Insert your second CCS term here $\bullet$ Insert your third CCS term here

\section{KEYWORDS}

Body; women's health; feminist HCI; biology and feminism; genders; embodiment; technology; 


\section{Introduction}

"What's a woman is a question that should remain open."

Judith Butler, 1992

What is a Woman? In present day, Merriam Webster's Dictionary defines "Woman" as a: an adult female person; b: a woman belonging to a particular category (as by birth, residence, membership, or occupation) [68]. Similarly, the same source advances a definition of gender as a classificatory term a: sex; b: the behavioral, cultural, or psychological traits typically associated with one sex (ibid). Gender, a social category imposed on a sexed body [90]; a representation, one which "pre-exists the individual based on conceptual and rigid (structural) opposition of two biological sexes" [57]. It is classifications as such that have strengthened the aforementioned dichotomy 'man' and 'woman'. This resulted in an uncontested understanding of biology as fixed and as destiny, one in which, for the longest period of time, there was no language for distinguishing sex and gender, and which has dominated within a Western context ${ }^{1}$. While this vernacular remains current, the English language is in the process of adapting to new cultural attitudes about gender [99], and the binary prevalent in the West is being disrupted. In academic terms, it is the emergence of queer theory and postcolonialism in the second half of the twentieth century that, at the same time as a third wave of feminism emerges, challenge the very category of 'woman' and throws into question the founding premises of its identity politics [39]. Where does this leave woman then, and why does it matter? In this paper we consider these questions, through the lens of literature and our own design-led research. We will argue that - despite the problems and tensions it brings - it is not yet possible to drop the category of woman. Not least due to the economic, political, and sociocultural inequalities that exist on a global level in terms of women's access to education and healthcare, and which impacts negatively upon lifelong outcomes. We start by examining the emergence of the category 'women', and the medical specialism of women's health.

There was once the belief that 'God gave men beards as ornaments and to distinguish them from women', the absence of beards in human females a confirmation of their less noble character [87]. It was not until the age of Enlightenment in the 1700s that 'woman', who up until then was considered a monstrous error of nature lacking male perfections of mind and body (ibid), emerges as anything other than having been born subject to men. It is with the Enlightenment that a newfound attention to the body and kindred scrutiny on sexual difference paves the way to establishing the binary man and woman, one that is based on the opposition model associated with designated female and male anatomy [37]. It is in sequence of this that a new reality would be introduced the following century, that of 'women' as a monolith entity. 'Women' as a singular object of study was born'. As a class of people, this classification comes into being at a time when there is a growing interest in the body. It is also a time in which social inequalities were being exploited to show how humans perform within the economy, and demonstrate how sociopolitical affairs differ between groups. Based on this, further classifications emerge according to age, race, and sex. It is in agreement with such taxonomy of people that the female body, classified as unreliable, leaky and disruptive, is seen in affinity with those of the marginal(ized): women are, consequently, withheld by their supposedly natural biological processes [91].

Devalued the body and designated women, it was a political-socioeconomic climate that, entwined in conventional biological and racial taxonomies, contributed to define the binary that positioned the sexed

\footnotetext{
${ }^{1}$ In her influential essay 'Gender: A Useful Category of Historical Analysis', feminist scholar Joan W. Scott directs our attention to the fact that many Indo-European languages have long adopted a third category- unsexed or neuter.

${ }^{2}$ As alluded to in "Nature's Body: Gender in the Making of Modern Science" by gender historian Londa Schiebinger, studies of women and expert knowledge was delivered by men, the only group who, at the time, had the right to higher education.
} 
body, male or female, as static, ahistorical and determinate. Moreover, in defining women and men in terms of one another also lead to identifying 'woman' as the 'other', further contributing to setting the female body apart in e.g. a separate branch of medicine [73]. This would later be assimilated into what we know today as women's health.

\section{The Language of Women's Health}

Through language, gendered identity is constructed [90]. Similarly, 'woman' is a consequence of language: a 'man-made' language, as documented in [94]. One could argue that such classifications may pertain to the imaginary, and therefore not having to do with the real [38]. What to do, then, about the category 'women'? Taking a more current approach, we look at third-wave feminism, one that embraces all types of feminisms and which at the core seeks to disrupt binaries, all embracing of multiple identities and consequent ambiguities and contradictions in standpoints [21]. It is within these conflicts and contradictions that multiple feminist views on 'woman' begin to diverge, some arguing that a gynocentric orientation is essentialist, others contending that gender queering makes women invisible all over again (ibid). It is a fact that one continues to be categorized as a female academic, a lady doctor, or a woman architect, just to name a few, and the view on this is that such categorization works in detriment of women by emphasizing difference. But ultimately, different from whom? The universal standard is in fact not neutral, as pointed out in [94], and the bias implicit in language that emphasizes that women, as a category, are different, also means that they are less.

Much can and should be said about gender(s), however this paper aims to focus on the gendering of women - 'woman's otherness', and how this has contributed to shape health and care. If, before, gender ideals contributed to shape scientific knowledge then the assignment of gender itself has been the result of the cultural production of a notion of what 'woman' is. We contend that gender is not a given on the basis of a given anatomy and that it is culturally situated [22]. That both gender and woman are concepts that are unstable and entangled with notions of identity, concepts that are more effective if challenged and refurbished, other than strengthened by repetition [80]. Nonetheless, woman's otherness brought about a new medical specialism, one that is carried on yet needs to be defined differently. In line with that, we strive to improve the design of interactions, devices, tools, and technologies that account for those that sit within these continuous meanings, as change needs to happen both in culture and in the existing apparati of mental and bodily health and care.

In this paper, we attend to the historical temporalities of women, to query woman-ness and that of 'being a woman' [60], through echoing Judith Butler and sustaining that "woman is undefinable and unwritable, where definition and writing are tools for the genericization of women" [18]. We acknowledge the gender gap produced with 'woman' to bring forward the historical oppression and disempowerment that effect the production of language and thought. While undertaking that producing women and women's bodies - as we may classify them - is critical and worth of study, we realize how this is rather disputed generally and within the field of research in which we work. While there is a growing interest in exploring topics that might be perceived as related to women's health, there is also resistance to this growing body of research. On the one hand because it may be perceived as essentialist and, on the other, in that it continues to be understood as a narrow field of interest to only a few. Topics that involve bodily functions such as menstruation are rightfully contested as a category pertaining to women's health or women's issues, both in academic research [52] inasmuch popular science [105], yet remaining a global human rights issue [112]. We are interested in understandings of how an agenda for change in health, one that attends to an historical 'otherness' and has shaped bodies and care herein, but also the lack thereof, can contribute to justice and alleviate inequalities. In thinking beyond empathy, we want to account for experiences in care that are dynamic rather than essentializing to highlight how and why women are still missing from the research, and how this lacking perpetuates othering in ways that also affect distinct groups of marginalized people. With woman-centered 
design we suggest to focus on humanity, activism, and inclusivity as approaches that foreground intersectionality, equity and equality. These, as entwined as they may be, can help achieve justice and break down the hierarchies that prevent everyone to access and participate in the production of knowledge that manifests in e.g. health care products, services and technologies. Following this, and based on our eight years of design-led research in women's health and intimate care, we contribute a methodological roadmap that others may use when approaching work in this complex setting, and illustrate and critique the research through design approaches we have applied in e.g., FeedFinder, Labella, and in design work in Lebanon with Syrian refugee women. We make the case for woman-centered methodological approaches to design, suggesting a pluralism that aims to include all and every woman, to propose woman-centered design as a methodology that supports and enables health care in women, while arguing for its wider applicability in, e.g. education, public policy, and, importantly, in practices of everyday life. HCI must consider its relation to institutionalized policy and practice to make change at a global scale. Nevertheless, doing so requires a critical understanding of what it means to be a woman, in historical and contemporary life, and how notions and concepts of woman and womanhood have impacted and limited the ways in which technologies have been designed. While a human-centered approach to design certainly intends to account for all humans, we see a woman-centered approach to potentially benefit humans who thus far, within human-centered computing, have been given less attention to or wrongly been deemed of interest to the few.

\section{$2 \mathrm{HCl}$ and the Body}

Technology and women withhold a history of avoidance. As argued by feminist science and technology scholar Judy Wajcman, the very definition of modern technology is cast in terms of male activities [116]. This is an historical viewpoint that infers that technology, as an area of male expertise and area defined by them, also serves to legitimize specific areas of concern. What is implied is that a woman's realm may be dismissed, as it is likely deemed unworthy of serious notice. In this sense, women are marginal to a malecreated and male-dominated technology. Exemplars that are relevant to our topic of women's health can be found e.g. in [46], a report showing how women are unnecessarily suffering and dying from heart disease, or the gender bias in clinical drug trials as discussed in [85], through mundane examples that expose e.g. data bias, shared in popular science formats such as [28][86] and which reach a wider readership. Within HCI more specifically, feminist perspectives of, and approaches to, what technology is, have contributed to gendering agendas within the field [36]. Still, within HCI, gendered demarcated technology has been criticized, particularly when it focuses on women, see for e.g. [82].

Indeed, the body is an access point for technology to enter and a medium to manipulate. The dissolution of boundaries between the body and technology - implantable, genetic, biologically enhanced, or contraceptive - are some of the impressions left by human (technology-abled) enhancement on personal, cultural, and physiological conditions. It is this profound impact that technology can have on any body that we focus on. Specifically, we are interested in the lived experiences of women within health and care, including the most subjective and beyond the stereotypical. In other words, we inquire how the de- and en-gendering of technologies could play a role in gaining, e.g., design knowledge of the body or, more generally, promoting positive experiences. While we approach this from a design research point of view, it is rather aligned with sex and gender research as seen in the Gendered Innovations interest group at Stanford University [113]. The group is interested in adding value (to research, engineering, society, and business) while stimulating genderresponsible science and technology. Researchers within the group mainly develop methods of sex and gender analysis for scientists and engineers to create new knowledge and technologies. 'Fixing the knowledge' is crucial, and de-biasing algorithms as much as en-gendering or de-gendering research in health care is critical, if not only because doing research wrong costs lives and money but it also poses threats for women (ibid). Acknowledging the need 'to modify technology to fit people, rather than modifying people to fit technology' [73] is not new. Many medical therapies have been designed specifically with and for the male body [88], 
and subsequently adopted by any body. While two sexes may not be enough to describe human variety [116], a renewed awareness of gender variables for health research can significantly contribute to ameliorate care within what we know as women's health and beyond.

\section{Gendered technoscience}

When attending to technology in relation to the body and/or women's health, we cannot ignore the wider realm of science. While technology differs from science in that science is about discovering and explaining and technology is about designing and making, one serves the other and vice versa in the pursuit of advancing people's quality of live more generally. It is in the health-related sciences that we find that when making a distinction and identifying 'woman' as 'the other' is what resulted in setting the female body apart in a separate branch of medicine (gynecology). It is with the emergence of this practice that women become identified as 'a special group of patients' [100], and that a professional expert practice, one that emphasizes the medicalization of women's health in detriment of women's traditional knowledge and helps establishing "women as objects of knowledge, but not as authorized knowers" [109], gains control. Moreover, and further compartmentalized within the broader categorization (women), it was this one-size-fits-all discourse that emphasized the universality of women and their bodies, a discourse which largely erased diversities [100]. True to this, as scholar Nelly Oudshoorn puts it, it was the very classifying of woman as the other that directed attention to the similarities among women. As a consequence of that, the design of technologies that attend to women's care within (and outside) the medical institution lack in adequacy or fall short when accounting for the diversity of its users. Nonetheless, it is true that a proliferation of women-centered technologies has taken to the market recently. Based on a business model that aims to empower individuals and promote selfcare, this growth of self-designated women's health technologies is making what is commonly understood as women's health related issues more visible. On the one hand, this shift in the commercial realm of womencentered products, i.e. 'female technologies' otherwise known as FemTech, is an attempt at fulfilling unmet needs. As a category, it refers to e.g. software, diagnostics, products, and services that use technology that focus on women's health [83]. One could argue that FemTech does bring to the fore issues otherwise neglected or considered unacceptable. Furthermore, these new or re-newed technologies promise to be accessible and reachable to most, are designed to be wearable or to be used at home, and playing their part mostly outside the clinic. On the other hand, criticism abounds, e.g. some claiming 'FemTech' to be sexist or that it disregards segments of the population. Does this industry ghettoize women, is it inclusive towards women, or does it condition experiences that pertain to varied genders? All standpoints may be valid, and what we see as resonating with our research is that, in fact, attending to the specificities of the body - a body that is e.g. leaky and transformable - matter. Moreover, technologies that promote self-care and which, to a certain extent, offer the individual an added or alternative way to manage their care and their conditions, might contribute to improve current healthcare systems by making interactions between patient and medic more sustainable, e.g. a change in physician-focused care to technologies that enable patients to self-monitor their health, or be more inclusive in that it introduces new tools for people or conditions otherwise overlooked or neglected. However, as women's health is going digital, concerns arise as the promise of such personalized care comes at the expense of women's privacy [61]. Furthermore, and while privacy and data protection issues are main concerns regarding FemTech mobile applications [83], they also risk to stereotype and work to configure new norms of behavior [62]. This could mean that they would not serve the woman at times when digitized data renders flat in its complexity the subjective individual experience, or that the quantified knowledge does not account for sociocultural conditions and therefore not fully accounting for the body, in its single and discrete geography, or embodied experiences.

Nevertheless, science is not a neutral culture when it comes to women. The systems of inclusion and exclusion from the communities that configure (scientific) knowledge have long conditioned how women know what they know [43][44][87]. It is not long ago that scientists sought to distance themselves from things defined as feminine, including women [87], and women as a group of people were ignored in the production 
of knowledge, even so when knowledge was directly related to them and their bodies [109]. As advanced elsewhere by Science and Technology Studies scholar Michelle Murphy, what is commonly called the women's health movement was "an example of doing technoscience differently, of technoscience as a popular counter-conduct at the nexus of oppression and enjoinment" [72]. Indeed, the women's health movement saw second-wave feminists taking charge of their bodies and contesting traditional and enclosed modes of producing knowledge. Decades passed by and the movement itself may be contested [86], i.e. lack of intersectionality in feminism. Yet, while lacking, its significance is lasting and continues to inspire. Whether to revalorizing the feminine or women's agency and capacity for empowerment, approaches within gendered technoscience ascribe value to the materiality of technology and how it affords or inhibits the doing of particular power relations' [115]. It is such materiality that we see critical in the design of e.g. new medical devices and tools, or when designing for the lived and ageing body. For example, in [3] we attempt to reflect on matter by exploring how enabling knowledge through material engagement can support conversations in tabooed topics that are implicated in the normal functioning of the body but also in its disruption, or how the bodily fabric can be a material of care in itself, e.g. by exploring the possibilities for designing within a symbiotic relationship between bacteria and the human body [107][108].

\section{A Woman-Centered Approach}

HCI design and research that is concerned with women's health as a topic remains underexplored or unattended to within field. In 2016, we reviewed women's health in HCI [5] and drew attention to the existing literature up until then, one which was mostly exclusive of technologies in maternal care. Surely, a few other topics had been touched on superficially. Our argument was one that showed how most (if not all) kept distance from the 'dirty work' that permeates the body, even if the research was ingrained in the messiness of the body. Following that, and in aiming to push the agenda forward when reimagining how the design of technology intersects with women's health, we proposed to engage with the HCI community through a twoday workshop at CHI 2017 [11]. Through this and what came after, we learned what we had already suspected was happening with much of our previous work and attempts to publish it: that the female body is contested (and political), designing for women's health is troublesome (and therefore not scientific?; prejudicial), that this mixing of female body and women's health is only relevant to the minority that makes up for half of the global population [12] (not representative enough?). That this hampers people in the field to work productively, is a whole issue in itself. Regardless, research emerges and a body of work is now starting to shape up. HCI researchers have brought to the foreground issues which had previously not been attended to before. Most recently (2019), these have included designing or not designing for menopause [15][51][58][111], intimate bodily fluids such as menstruation [122], chronic pain syndromes e.g. vulvodynia [126], or conditions overarching women's lives more generally [100], just to name a few. For the most part, these make available a wide range of possible methods and approaches to advance or speculate on the possibilities and challenges of designing within the area, as broad as it might be. This work may continue to be scrutinized at a higher standard, as mentioned in [12], and researchers may find themselves at a disadvantage because of the nature of the research they engage with [119]. But as issues of gender unfold and are attended to within these academic venues, they add to the broader conversation [23]. In wanting to promote equity and inclusivity within the field there are many qualities of being a human we need to account for. Our pursuit to design for and with woman aims to be inclusive. We hope to contribute to a much needed conversation that, in inquiring woman-(other)ness in relation to e.g. the process of othering in the (bio)medical sciences and, subsequently, in the design and use of technology, problematizes woman in ways that are helpful in regards to making the field a better place for all.

\section{A Woman-Centered Approach in Design Research}

Within the broader design research academic context approaches are emerging that are to some extent aligned with reimagining the body. For example, in the Design Research Society International Conference the Design, Research and Feminism(s) track new to DRS2018 [67] emerged as a consequence of discussions 
held in previous editions. It aims to highlight situated knowledges, those that may entwine and advance e.g. 'feminist perspectives on knowledge-making', or feminist design and affiliated practices. Reframing design problems within women's health [50] was one such paper presented in one of the track's sessions, and which reflected on the complexity of what it means to be a woman in today's society. In line with a renewed interest in understanding such meaning(s), and integrated in the program of Conversations adjacent to the conference, we conducted a Conversation titled Woman-Centered Design [7]. In doing this, we set to explore how the design of technologies and interactions can act critically in the ways that they serve, refigure and redefine women's bodies in light of what woman is, opening up to the challenges and opportunities of designing for and with women. Much like the workshop at CHI [11], this Conversation invited participants to reimagine approaches and technological apparati in relation to women and their health. Similarly, the aim overall was that such a hands-on approach could contribute to opening up future discussions and to involve others whose research and/or practice may be intertwined with our quest(ions) to produce knowledge that enables a myriad of design approaches that serve as positive paradigms towards all women [7]. While thoughtfulness, power, biotechnology, beauty and disgust could somewhat characterize the main themes emerging from the Conversation, with this paper we seek to make a more targeted contribution to the broadening of discussions around the body and women's health in HCI.

In doing this, we continue to engage with aesthetic and critical approaches to bodily and women-centered experiences, which are pivotal to this research. We are of the opinion that bodily autonomy and selfdetermination of gender are basic civil rights and that notions of authenticity in relation to gender constructs pertain to the imaginary, as argued in [38]. Nonetheless, it is a theorization of gender roles that has established less than imaginary hierarchies and it is within this space that we assert the value of e.g. women's health. No doubt, the universal category of 'woman' might be replaced by human bodies in all their diversities [73], and the methodological roadmap we propose in this paper hopes to show just that. We propose woman-centered design as a way to move forward, if at least to inspire and defy the current, ongoing climate related to women and their bodies. To do this, we root our approach in feminist theories of technology [116] and technoscience e.g. [9][38][66], entwined with critical-humanistic HCI as proposed by Bardzell and Bardzell in [14]. While it has been argued that designing for women risks ghettoizing them [24], or that a risk lies in designing technology explicitly for women [82], we will place an emphasis on approaches that embody lived experience, but that also position approaches to a woman's health as demanding of justice, in that it regards bodily integrity and takes women's rights into perspective. We introduce women-centered perspectives and empathic approaches to health by placing a woman's body as a lens to relate to possibilities of future technologies for care. To this end, our roadmap starts by raising the stakes of established approaches within the field, e.g. empathy.

\section{Thinking Beyond Empathy}

"A woman-centered approach to health is a human rights-based approach that seeks to ensure that every individual has access to basic health, education, and other social services, including sexual and reproductive health"

US Global Health Initiative (2010)

At the time of writing, there is little doubt that the approach mentioned herein (2010) is heavily disputed under the current US administration. However, and while we write from a Western European context, the piece of public policy we refer to was instrumental at a time when we started designing our own research. That women's autonomy in health is scrutinized at policy level is a reality that many started fighting against decades ago, and many continue to today. In our view, policies that are detrimental and have the power to deteriorate life and are dangerous to a person are not helpful or a step towards providing people with a life of dignity. 
It is true that scientific inquiry and thought have produced problematic, inaccurate, false, and even harmful understandings and "facts" about men and women [56]. And that some of this 'scientific knowledge' needs to be revised and updated accordingly. Moreover, in taking that technology results from the combination of artefacts and social practices [53], and our focus being on the social institution and systems of knowledge, it is critical that advancements within adapt and conform to contemporary knowledge of the body. No doubt, knowledge of the female body has changed considerably since the 1800s. One significant change made visible in medical language was that to linguistically distinguish 'organs that had shared a name, ovaries and testicles... organs that had not been distinguished by a name of their own, the vagina, for example, were given one' $[37]^{3}$. It is worth considering, i.e. particularly in practices entwined in health and bodily care, that such acknowledgement in language denoted that body parts are different between them, or that they require welldefined examination. Moreover, the existence of gender bias and disparities in women's health and care occurred due to the fact that "biomedical research findings on women's health issues were based on male subjects and later generalized to women" [71]. While stereotypes permeate notions of women's bodies, by perpetuating generalizations of the body from men to women which hinder the (ever-evolving) field of research and knowledge known as women's health, so does attending to human bodies in all their diversities becomes more than an imperative requirement. This will involve transformative acts of and empatheticcentered approaches that can challenge existing hierarchies of sex and gender. As much as language evolves, so does the living organism that is the body. In modern day, the body is multiple.

Empathy foregrounds embodied knowledge, affective connection and a desire to transform the social terrain [48], in a way challenging the experience of our own sense of being and the experience of possibilities and limits to how we may act or be (ibid). Empathy and experience in HCI have been extensively researched by McCarthy and Wright in [123], with empathy understood as a necessity in moving from a focus on system functionality to a more humanistic perspective on design (ibid). As argued by the authors, an empathic approach extends to include and build on inspiration achieved from a rich understanding of people's experiences and life contexts, with the purpose of developing "through a meaningful emotional encounter between designer and user". An empathic methodology carries through after fieldwork and engagement with the participants to give expression to participants' experience in the analysis. Nonetheless, the slip between empathy and pity has been acknowledged to take place in such contexts, and to weaken what empathy requires [48]. In light of this, we pursue research that, in designing for women and their bodies, specifically within the context of women's health, is disengaged with ghettoization and rather it is critical to redefine and rescript individual bodily experiences of and in care. The gendering of technologies can be understood as not only shaped in design, but also shaped or reconfigured at the multiple points of consumption and use [116]. We are interested in 'artfully integrating' design practice and its objects in ways that may reconfigure its use, mostly by women but open to all genders, and with women's rights in mind. In wanting to engage with aesthetic qualities and a critical viewpoint to bodily and woman-centered experiences, and in exploring material and physical engagement, we aim to advocate for feminist-oriented approaches, to designing technologies and promoting knowledge that improve the conditions of all women. In what follows, we elaborate on three approaches that emphasize and imply empathy but which also look to break down hierarchies, and that we see pivotal when designing for and with woman: humanistic, activist, and inclusive.

\section{Humanistic}

Designing human-technology relationships with a humanistic approach encourages an openness to novel ways of thinking that can support research in e.g., sensitive topics and challenge lines of inquiry that may be absent or even 'forbidden'. As human-centered perspectives in HCI have grown to embrace "concepts such

\footnotetext{
${ }^{3}$ Interesting to note that linguistically some continue to lack words for genitalia e.g. the vagina: that part of the body does not exist in Burmese [65].
} 
as aesthetics, user experience, enjoyment, and play" [13], these approaches have helped place meaning within "understanding of people, their concerns, and their activities at the forefront in the design of new technology" (ibid). In doing so, a humane approach acknowledges the desire to reinvent the status quo of humantechnology relationships and suggests a shift in focus regarding social, cultural, and political concerns and actions. Furthermore, critical-humanistic approaches to HCI account for research concerned with designing interventions and interactions for everyday life, "including how they fit in technological, cultural, and environmental ecologies from the beginning to the end of their lifecycles" [16]. Also, there has been a shift as a result of humanistic and feminist approaches to conduct intersectional research. In challenging gender more generally, or specifically in its relation to the design of digital technologies and the impact of computing on people and power structures, third-wave HCI has previously called for intersectional approaches to research - such that account for gender but also ethnicity, race, sexuality, or class - to promote equity and inclusivity in HCI [119]. A deeper engagement with identity complexities has been summoned in recent research [89], and is visible in recent research that discusses e.g. safety for transgender women and nonbinary people of color [96], identity on social media [41], or trans-inclusive design practices as put forward by Ahmed in [1]. This roadmap attends to the "capacities of digital interaction as a medium" to design for and with women, while "explicating relationships between design choices and experiential qualities" [14]. In advancing an approach to designing experiences, tools, services and interactions that serve women specifically, we aim to engage humanistic approaches to the body and digital as mediums. Within, there is a commitment to approaches that are intersectional and that ought to address women in their diversity.

\section{Activist}

Obtaining justice in women's health care became eminent with the women's movement originated in the United States during the late $60 \mathrm{~s}$ through the early $80 \mathrm{~s}$. Associated with the second-wave of feminism and known as 'the women's health movement', it contributed to bringing to the foreground inequalities as varied as reproductive freedom to pay equality in women. Moreover, the impact of feminist knowledge in, for example, gender bias diagnosis - which most commonly occurred in relation to the reproductive system -, had already seen a push towards medical knowledge that reflected women's reality [70]. To that sense, it was also at this time that the 'medical industrial complex' was counteracted by nonprofit alternatives such as women's health centers or free, community clinics [72]. The disentangled relationship between governmentality, the political economy, and the people it is serving continues to this day, and lead to the growing on-the-ground projects that we hold here as activist. E.g. in terms of bodily health, i.e. in reimagining medical devices and products, through the de-medicalization of experience, approaches and methods that utilize e.g. data are well positioned to challenge hierarchies and in turn support the dismantling of existing systems that abide by established power structures; or citizen-centric initiatives that promote knowledge, share resources, and are informed by feminist approaches to technology and society-oriented issues more generally. Within HCI more specifically, researchers have discussed technology as a means to understand e.g. health inequalities in relation to social determinants of health that impact people's livelihood [104], or explored how building community capacity can benefit a healthy lifestyle [75]. HCI accounts for a growing body of activist-driven research, including inter-transdisciplinary explorations and directions that involve health and the body, e.g. [54], gender [95], empathy and food [33] and more broadly, health activism in HCI, e.g [104]. In other words, challenges are real and the opportunities for change are immense.

\section{Inclusive}

We consider design and how it can be an effective tool for social change as much as it can be a tool for oppression. In regards to positive impact, its "peculiar, fluid position as a discipline capable of benefiting from both humanistic and scientific knowledge has long been one of its most distinctive traits" [76], as much as material objects can be social agents [74]. In [76], Prado de O. Martins questions the relationship between gender, technology and socio-cultural oppression, such as gender violence and discrimination, to critically 
argue existing constructs of gender stereotyping. Stereotypes, as we mention before, have never contributed to an agenda of gender equality. Indeed, stereotypes about women's nature and women's bodies - disputed as they may be, more so as we move forward in waves of feminism - have played a part in constructing knowledge throughout the centuries. Said knowledge has impacted health and care within professional and clinical encounters but also on personal and intimate settings. That is to say that designing for human bodies in all their diversity contribute to de-center and overcome the one-size-fits-all discourse. Our aim is to resist any universal point of view when producing our designs and the diverse subpopulations we have worked with or others we will work with in the future. Pluralist designs, as advanced by Bardzell in [17], are likely to be more human-centered than universalizing designs, and it is this decoupling that makes space for perspectives that are sensible to marginal or marginalized individuals and communities to emerge. Moreover, as noted in [59], researching the needs of a subset of a larger population can still involve underlying needs that a more general group or other subgroup can benefit from. One example could be that of a technology that traditionally has been designed for women: fertility tracking. This is a technology that targets a large number of the human female population, and in redesigning it for a shared-experience can make a difference to a significant number of men too [52].

We recognize that affects and effects of oppression(s) cannot be understood independently [35][89][119][120], and that equity and inclusivity across intersections is critical to advance a practice that is responsible and equipped to make a valuable difference in people's lives. From designing for and with underserved communities [34] to 'lighter' issues such as "the recent wave of unnecessarily gendered products" [76], technologies are both positioned to empower and disempower in distinct ways. While gendered relations are certainly among the social relations that artefacts embody and convey, we are interested in exploring methods that are oriented towards making a difference while addressing specific problems, and the capacity that emerges from the use of that method to change the problem [63].

\section{Doing Woman-Centered Design}

\section{Woman-Centered Design as Inquiry}

In recent years, an increased number of design practitioners within $\mathrm{HCI}$ have contributed to the merging of practices that employ a variety of methods and conceptual viewpoints in what, we would argue, includes critical, inclusive, activist, participatory and speculative approaches that implicate women, their bodies and a wide range of lived experiences. As a team of researchers contributing to the field of digital women's health we have worked across a multitude of topics and contexts. We have conducted this work with one another, and with a number of other colleagues across a range of institutions in the UK and worldwide. These projects have been imprinted with notions of humanism, activism and inclusion to greater and lesser extents. For example, [69] describes a field-based, participatory practice that involved women in discussing experience, and policy (making) in Ireland regarding abortion rights. Working with the pro-choice community within the country the research team engaged in activism through exploring the method of digital storytelling as an approach which could generate 'stepping stones' for activism. In putting forward such participant-based methods of engagement, narratives and realities the team aimed to highlight and support individuals on the ground in 'breaking the silence' around abortion, and having conversations at a community-level which would stimulate the Irish voting public to vote for legalizing abortion. In [98], the research team took a humanistic perspective to investigate how HCI practice and research can overcome the live endangering stigma associated with sex work. Principally by identifying sex work as a legitimate form of work that an individual can choose rather than the stereotypical view that sex workers are forced into such work. In this section, we critically reflect on three long-term and ongoing projects which have employed a woman-centered approach to design. We aim to consider the ways in which these projects are humanist, activist and inclusive, while also highlighting where our approach has fallen short of these goals, and our learning as related to these shortcomings. 


\section{Study \#1. Access to Embodied Intimate Knowledge}

Labella is an augmented system that invites the woman to be an active participant. In this specific case, she participates by looking through an interactive surface (a mirror) to the body (the vulva). The system combines a pair of underwear for embodied intimate interaction, and a mobile phone as a tool for embodied discovery, and it has been thoroughly described in [6]. This proposed an unconventional tool that works in the interest of generating knowledge that is actively produced. Specifically, it did so by considering biological embodiment as part and parcel of what makes bodies what they are, it aimed to draw attention to the 'scientific' intimate body and particularly to address 'invisible' parts such as the pelvic floor muscles, in order to highlight pelvic fitness in women.

Labella as Humanistic: We return to knowledge, or lack thereof, as actively produced. We revisit this notion when considering methods that we have used in our work, e.g. that involve design workshops with communities of women ${ }^{4}$. We want to not simply provide women with knowledge but to involve them in the production of, and in developing new, knowledge about themselves. In the case of the eTextile Toolkit, which preceded Labella, we created a textile-based toolkit that combined aesthetics and materiality to explore notions of the intimate body. This toolkit was an invitation for women in a series of design workshops to recall existing body knowledge and further explore bodily knowledge through crafting and technologyenabled materials [4]. Other than simply consult medical textbooks to obtain information and understand the intimate body [109], women were encouraged to become embodied knowers and engage as active participants in the processes within and between their bodies. We see this research as contributing a designerly approach to creating bodily awareness through hands-on engagement with crafting technology. Furthermore, the design workshops were a field site for them to work and learn collaboratively in, one which would enable knowledge through material engagement. It is such engagement with textile-based materials that offers opportunities for advancing understandings of the biology of the body, in this specific case that of the inside of the body and physiological processes involved in pelvic health, i.e., the pelvic floor muscles, body organs such as the bladder, and bodily fluids, e.g. urine, implicated in continence care. It is based in the experiences with this toolkit and series of workshops that we then created Labella, which preserved the physicality of the interaction, through extending to digital design e.g. the expressions included in language as used by the women to describe their bodies. Indeed, using language that is culturally available - and employing it as means to both re-member and (re)learn the body - served to push towards what is more contextual ways of explaining these biological processes. As feminist biologist Lynda Birke notes [20], "by ignoring bodily insides we run the risk of perpetuating the biological body as fixed and presocial - even when that is apparently denied by arguments that we cannot understand our biological selves expect through culture". An understanding of the biological body which allows for dynamic processes, that is transformable, is a move away from notions of fixity and constraint. It serves to create value within the embodied experience of those - through Labella, cisgender women who participated in the study - whose voices have been absent or have not been heard through the establishment of e.g. medical ideologies. How bodies work are not culturally neutral [20], and we acknowledge that representations of bodily out/insides as seen in and with i) the toolkit and ii) Labella, are a reflection of the culture in which they have been developed. Nevertheless, these were research driven, working prototypes whose goal was mainly to ignite a discussion around the 'embarrassing theme' of the biological body - one that is transformable other than determining.

Labella as Activist: Conceptually, Labella is inspired by the women's health movement, as introduced before. This was a time in which women used a mirror as a technology of choice to not only highlight health

${ }^{4}$ We acknowledge that not all women have a vulva or may have a different anatomy from the one we designed for, or that people with a vulva and said anatomy might not identify as woman. This research focused on those who are most readily defined by these

biological processes and biological body, and who may suffer because of it. 
rights (or lack thereof) but foremost to reclaiming control over their bodies and challenging political power. Women "located power in the mirror and in genital self-visualization, and they reclaimed both as productive tools with which to confront the male-dominated institution of gynecological medicine" [55]. The mirror provides a means of outer representation and, in 'going through the mirror', the woman in Labella is invited to explore her body in a non-traditional way, one in which the body space merges with a sort of representation(s) on the screen. By doing this, we aimed to explore technology as an extension of the body, one that invites the senses of (visual and tactile) representation to operate in tandem with the physicality inherent to the interaction. Looking at oneself, as requested by the experience with Labella, allows for creating a deeper bodily integration. Looking, however, is based on the decision of the woman. It is the woman that starts the interaction and has control over it, and all this is happening while exploring external and internal body organs, which relate to specific bodily functions. By focusing on self-care and the pelvic floor muscles we respond directly to challenges some women experience in weakness of the muscles group as a result of childbirth, menopause or obesity. Such weakness can lead to urinary incontinence which in turn gives way to medical interventions that have been found to result in extreme pain and further internal damage. Some examples include pelvic organ prolapse, e.g. bladder, uterine or vaginal, a condition where organs fall down or slip out of place, and which, ultimately, require surgical management. Recently, news press have uncovered the many complications associated with such mesh surgeries, from accounts of women and families affected by them [29][30], through the fact that these longstanding interventions have been standard practice without clinical trials [31][32]. Thus, within this context, we see continued self-care of this muscle group across the lifecourse by women as an act of self-reliance and a rejection of medicalized intervention and experimentation on the body. Further, by familiarizing woman with her anatomy, providing a language to describe her body, we reduce the challenges that woman might face when attempting to identify and care for issues she may be experiencing. Moreover, Labella was also experienced within (heterosexual) couples to enquire how knowledge, of not only the self but also the other, promotes capacity in an intimate partnership [2]. Importantly, this particular study showed that 'experiencing together' invited into conversations that otherwise would not have happened, e.g. one couple found out one of them was doing pelvic exercises which the other was not aware of (this due to awareness of the exercise's benefits in relation to ejaculatory dysfunction), and that caring is two ways, e.g. learning about the other is a medium to care for an intimate relationship, and that understanding the benefits of pelvic fitness is advantageous for both sexes.

Labella as Inclusive: The woman produced through this design is a woman who has female genitalia, whose genitalia are neat, and hairless, and who is flexible and slim enough to engage in the contortion required to place a phone between their legs and still view the screen. Overall, the design of the female perineum as seen on the screen shares much with the existing traditional resources of 3D anatomical models one would see in an expert clinic (used to e.g. show the reproductive organs or the anatomy of the pelvic floor, as we have discussed elsewhere [5]) or anatomy books. However easy to identify by some, and while representative of a people's anatomy, these body organs are non-specific to the individual (one-size-fits-all approach to learning). While we aimed differently with Labella, in regards to approach to learning, this first prototype included visuals that were similarly standardized. Moreover, bodily awkwardness and indeed awkwardness of core-body movement is key to the experience with Labella, which may put some bodies more at ease than others (one participant in the study suggested the use of a selfie stick). We aimed to explore a naturally 'hidden' part of the body that is not only physically concealed but also concealed from body knowing. Built into this, is a design interaction that speaks to some.

Labella was used by a diverse group of individuals who identified as woman. Some were experts in physiology and others had a mere basic education of their own physiology. For some of our users Labella provided a fundamentally different view of the body: a feeling of being permitted to look at their own vulvas; the discovery for one participant of her clitoris; and for other participants a more embodied understanding of how to care for the pelvic floor muscles throughout life [6]. As we argue earlier, this development of an 
individual appreciation of the body is arguably central to the demystification and appreciation of a woman's body, providing collective strength over time to challenge the social-cultural norms which lead to gender discrimination and violence. At the time of its presentation at ACM CHI 2016 Labella received several critiques which highlighted areas that lacked inclusivity and diversity in the use-cases and representation of the female body and which could lead to stigmatization and further oppression of others. First the hairless vulva within the prototype suggested a very particular view of a woman's body, which many did not see as reflective of their own bodies, but rather an idealized woman's body under the male gaze. This very stereotyping of the vulva and its 'neat' and idealized appearance was not intended to be communicated by Labella, but it was an unfortunate artifact of the particular constraints we experienced as we developed Labella. Clearly, a future iteration with Labella would include redesigning the prototype to accommodate both differences and similarities, and in that way to not merge the vulva to one tight configuration; to expand and include representations of the number of disorders that may affect the vulva (diseases of the vulva include e.g, inflammatory conditions such as lichen sclerosus or vulvar psoriasis, through vulvar cancers, most common being vulvar squamous cell carcinoma and vulvar melanoma). Furthermore, our focus remains on pelvic fitness, and for this we would continue to explore ways to 'exercise' the complexity of the pelvic muscles in health and disease.

The interaction with Labella was designed and experienced as 'awkward', both socially and physically. However, as a physical design the ergonomics of the interaction with Labella meant that some bodies were less able to view their own vulva. The design of Labella privileged bodies that could be flexible or were slim enough that the view of the device was not impeded. Other individuals could find ways to view the screen, for instance by placing the phone on a table or using a bed, but this further distanced them from the interaction and their engagement with their body. We sought to provide woman with an encounter that would encourage a humorous and slightly awkward exploration of the body, but despite this our design work made this exploration less available for some, with the potential to further stigmatise those particular bodies.

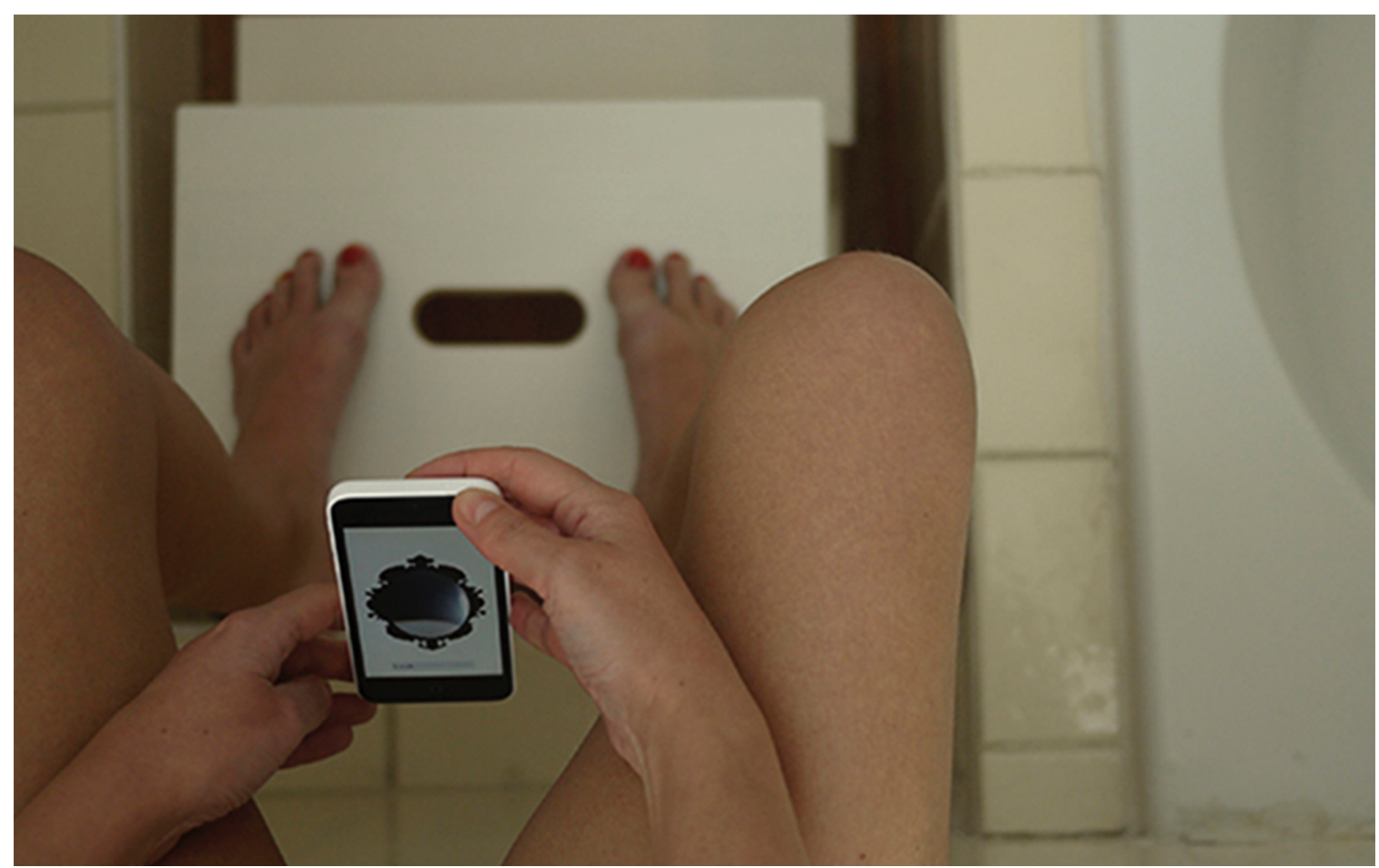

Figure 1: Labella, view of mobile app (screen 1: 'Look down there'). 


\section{Study \#2. Public Breastfeeding in the UK}

FeedFinder supports breastfeeding women in finding, reviewing and adding locations for public breastfeeding [10]. It is a project started in 2013 in the UK, and it continues to be supported, used and researched. It currently has around 8000 users, with well over 5000 places for public breastfeeding reviewed and mapped in the UK and worldwide. In itself it is a unique and open dataset describing women's experiences of breastfeeding in public. By proposing an alternative approach to finding places to breastfeed in public spaces, it contributes to empower members of the community. Presumably, they share similar concerns, and with this application they can add their knowledge to the issue by reviewing locations on a mobile app. While women are reliant on members of the community to leave accurate reviews, it is also an example that demonstrates how a work of social computing benefits a personal, individual act, while enabling a community of women as activists for personal wellbeing. It is an exemplar of possible discrete activist approaches for and with technology, one that can have a profound mediating effect on the way breastfeeding in public can become more acceptable or normalized the function and fact that is necessary for providing quality of life in both newborns and mothers.

FeedFinder as humanistic: Infant feeding is often politicized and associated with issues of class and race. Within the UK - where FeedFinder originates - breastfeeding is viewed as a middleclass concern. Nevertheless, due to the evidenced health benefits of breastfeeding efforts are made within public health policy and practice to improve breastfeeding rates across the country, and particularly within lower socioeconomic groups. Oftentimes emphasis around improving breastfeeding rates has been aimed at the individual woman to accept breastfeeding as an infant feeding choice. FeedFinder takes a different approach, recognising that the choice around whether to breastfeed or not is also highly informed by the particular socio-cultural context in which a woman lives. Through women's use FeedFinder collects a novel dataset which describes location-based experiences of breastfeeding. Our own varying analyses of the FeedFinder dataset have exposed the socio-cultural lines upon which the breastfeeding experience in the UK is composed [26][93]. In [93] we show how woman often describe resounded positive experiences of public breastfeeding (in deep opposition to the media narratives which continually describe deeply negative and shameful experiences of public breastfeeding). But, we also note the emphasis woman place on 'privacy' and discreteness in their textual reviews. Digging into this deeper in [26] we show how woman's concerns around public breastfeeding are intertwined with their physical location. In particular the data collected by FeedFinder has been able to show the stark contrast in experience and concern as dependent on the socioeconomic status of those areas. Thus, by providing a platform through which the lived experience of breastfeeding can be collected based on location, and 'in the moment' women are able to build a knowledge set grounded in their own lived experiences through which they can describe and evidence inequalities in access to breastfeeding and support for breastfeeding, thus making these concerns real for policy and practice. What has been harder, but what we continue to work on, is making this dataset available for action by public health officials, businesses, and health workers.

FeedFinder as activist: FeedFinder configures the woman not as a consumer but as an active participant and critically one who can contribute toward a supportive breastfeeding context. Thus, by providing woman with the tools to collect and view data about the breastfeeding experience in their local area woman can inquire about the 'friendliness' or acceptability of breastfeeding in their local context. Further, FeedFinder provides woman with an opportunity to collect data and take action based on their lived experience of breastfeeding in public - for example, as we describe in [10] a breastfeeding activist used FeedFinder to improve the breastfeeding support offered by a large retailer in her local area. But, we recognize that breastfeeding women cannot be solely responsible for changing how society views the act of breastfeeding. As such, the research team has worked extensively with local councils, community breastfeeding support 
workers, and the National Health Service in the UK to make the data describing women's experiences of public breastfeeding available to support the development of breastfeeding support practices and policies at a local level [92]. Throughout the project we hoped to configure woman as a voice which should be listened to, and whose experiences are worth considering in the development of policy and practice. In attempting to work with some local councils we encountered an unexpected opposition to FeedFinder. Occasionally public health workers described FeedFinder as undermining legislation which ensures women's rights to breastfeeding anywhere in the UK without interference. Taking the view that FeedFinder is prescribing a limited number of places where a woman can breastfeed, rather than offering insight into other women's public breastfeeding experiences. This critical view of FeedFinder was surprising, and not something that we intended through its design, nor encountered as we were designing FeedFinder.

FeedFinder as Inclusive: When we initiated the project our primary user for FeedFinder was the breastfeeding woman. We wanted to provide a resource which would support a woman in investigating the breastfeeding friendliness of her local area in the early days of breastfeeding so as to feel more confident about breastfeeding in public. As a consequence, we worked exclusively with those who self identified as breastfeeding women and who chose to attend public breastfeeding support groups in the areas of Newcastle and Gateshead (both of which have some of the lower breastfeeding rates in the country) to design and develop FeedFinder. What we did not consider in our design work is that FeedFinder was not solely used by breastfeeding women, but also by a breastfeeding woman's partner. This additional primary user emerged through subsequent interviews as we tried to understand people's experience of using FeedFinder. Reflecting on the design of the application - particularly with this broader notion of a 'primary user', and how FeedFinder is actually used - it is unfortunate that pink is a predominant colour in its design. We recognize that this stereotypical gendering in FeedFinder's design is likely off-putting to many of its users and potential users. Finally, we do not consider FeedFinder to be neutral, since its goal is to support the uptake and continuation of breastfeeding. Nevertheless, the rated qualities requested in a FeedFinder review are likely as relevant to bottle feeding as breastfeeding, additionally, there is nothing within the application which describes the benefits of breastfeeding over bottle feeding, or attempts to persuade women to change their infant feeding choice.
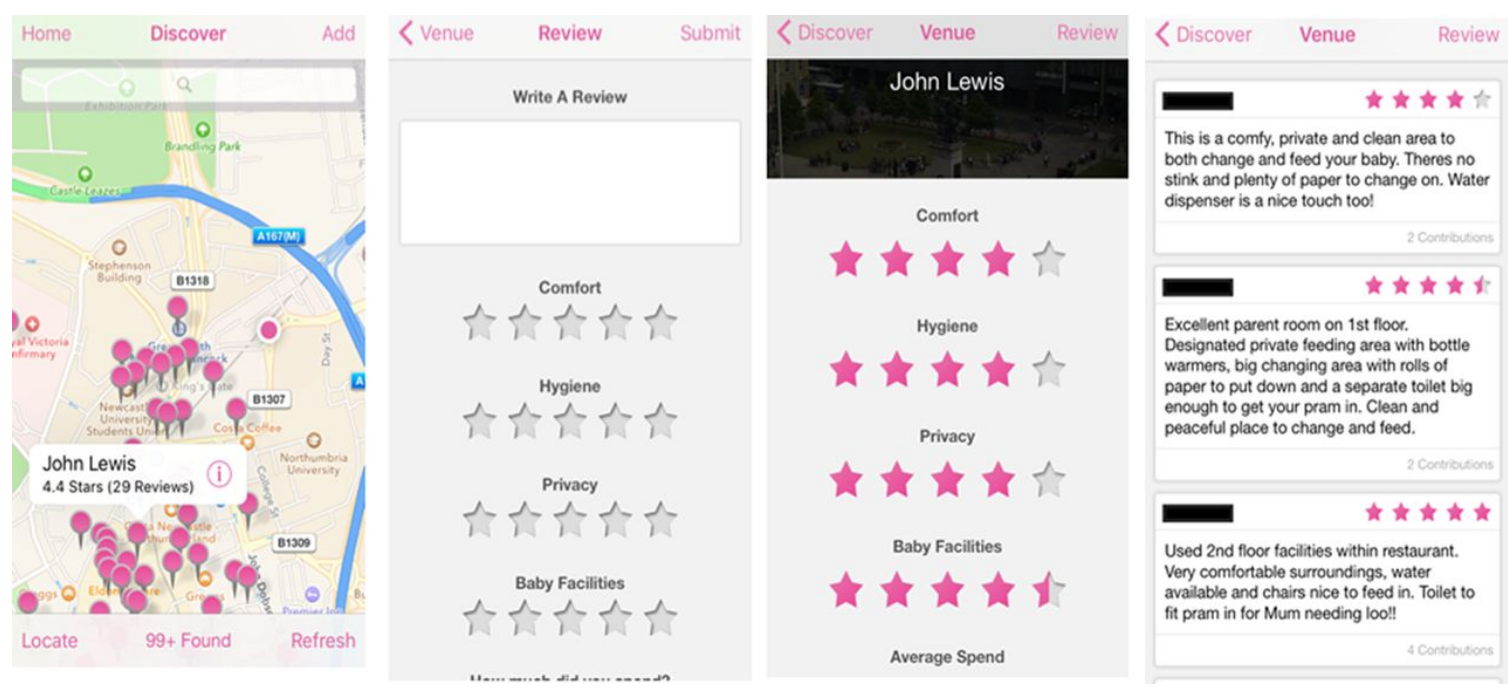

Figure 2: FeedFinder mobile app.

\section{Study \#3 - The Health of Refugee Women in Lebanon}

The final project we reflect on here is ongoing work with refugee women living in settlements in Lebanon [103]. Collaborating with several communities the research team has explored how design and technology 
can work to overcome some of the challenges that these communities face, from access to antenatal care [102], through to living with food poverty [101]. In contrast to previous case studies this work is based outside a western context, and broadens our concerns within 'women's health' beyond the biological to social (in this case food poverty). Unlike the previous two case studies this work has not resulted in one specific interaction design, but instead a series of different prototypes and design processes. This research contributes to the ongoing debate in HCI and e.g. health activism, in which sub-communities are interested in policies implemented by the government and which affect the health of already marginalized communities.

Humanistic: In one of our first explorations with this community we investigated how a synchronous voice interaction technology could be used to enable the women in the settlement to have real-time telephone conversations with healthcare professionals regarding antenatal care [102]. Within this particular context women in settlements often find it extremely difficult and costly to attend healthcare clinics, and view the support they receive when they attend these clinics negatively [103]. Working with the women the research team designed something akin to a community radio show where women would first receive some healthcare advice from a healthcare professional, and then following this would be able to ask and discuss a series of questions of the healthcare professionals that were important to them. Thus, similar to FeedFinder, the interaction design here sought to shift the view as women as passive receivers of healthcare, to active participants in the construction of knowledge about their bodies and their healthcare. Further, by establishing longer-term connection between healthcare professionals and the refugee women the design work impacted on the nature of the relationship and stereotyping of the various actors. Healthcare professionals established a more empathetic understanding of the women's situations, and the varying reasons why they may not be able to comply with healthcare advice, and the refugee women established a greater sense of trust in the healthcare workers, and a feeling that there was value in seeking healthcare advice in Lebanon.

Activist: In working closely with two communities living in permanent settlements around the topic of food security it became clear that the women were becoming increasingly critical of their previous work with researchers because they found they benefited little from participating in their research. Thus, as this work progressed the research team focused in working with the women to design and produce an artifact that would support the community in producing and reproducing knowledge which would allow them to care for one another. In the context of this project, this was a booklet that described the impact of austerity on their dayto-day lives, as well as a means for sharing their experiences. Throughout this research the women worked together to produce content in their own words, and with their own artwork that depicted their struggle to live in Lebanon given the country's current policies and practices around labor, housing and aid, as well as to provide support and guidance to new refugees entering Lebanon. At the women's request this was produced both as a paper-based booklet, and an online pamphlet. They intended to use this resource to help them to communicate their needs better with NGOs delivering aid, as well we provide guidance and support to refugees when they first arrive in Lebanon.

Inclusive: The focus on women was not an a priori decision, but rather a result of the cultural and practical context and limitations within which the study occurred. This was partly because men were working during the times that the researcher was allowed to access the community (due to lone researcher safety concerns) and as such it was the women in the community who were invited to participate in the research projects. But, the decision was also because some of the subjects that were centered in our inquiry (first, antenatal care; and then food security) were considered women-only subjects by the communities we worked with. By respecting these communities, and allowing the communities to decide who participates in the research, the projects put the participants wishes first and foremost. However, it is also fair to say that by respecting these wishes the research projects perpetuate the view that antenatal care, and food are women's topics within these communities. And, while working in a western context the team might feel obligated or able to challenge 
these stereotypes of ownership of knowledge, this is not something which was possible or feasible for the team to challenge in this setting.

The continued and prolonged use of surveys and other data collection tools by researchers on these communities was viewed as oppressive by the communities the team worked with, to such an extent that the communities began turning down the offer of 'participation' in research projects because of the lack of outcomes for the communities. In contrast the lead researcher of this programme of research approached undertaking this research as a process of collaboration and cooperation. Simple choices, like to sit on the floor with participants, to allow the women to 'pamper' the researcher by having them apply henna, or attend to her unruly eyebrows all served to show the women the humanity of the researcher, and her view of these women as equals [101].

\section{Discussion}

Through doing woman-centered design we have tied our projects to three core ideals, that of humanism, activism and inclusivity. By doing this within our projects, we have attempted to figure women as active participants and embodied knowers of their own bodies and health. This has been only a partial journey. In the following sections we turn to ask where we have got to on this journey thus far, and where we are going. As we reflect on what we might achieve with woman-centered design, we pursue the following three core questions: do our designs help women to better understand themselves?; does our design work advance equity?; and to what extent does our design work seek to reduce disparities? For us, these questions reflect an approach by which we can evaluate our work both formatively and summatively. And while qualitatively different, for us they function much as concepts of increased usability, learnability and error prevention functioned as a means for evaluating user centered design - as a way of measuring and reflecting on whether our approach is leading our design work in the right direction. In that, we hope to use these questions to help us reflect our own projects as they develop, to consider the kinds of impact that we desire to have.

\section{To better understand ourselves}

To better understand ourselves we have explored how particular design methods and design work can involve women in the processes of, and lead to, women becoming active participants in their care and embodied knowers. These have included creative approaches to talk about bodily taboos, e.g. Labella, or reconfiguring relationships between vulnerable communities and those that assist them in their health. Broadly, these approaches aimed to encourage openness toward novel ways of thinking about what might be perceived as e.g. sensitive topics, and to promote critical thinking in practice-based research while developing new tools and techniques for future health and wellbeing.

We understand woman-centered approaches to design as crucial to designing with care in the context of women's health but also as speaking to humanity, activism, and inclusivity beyond traditional humancentered approaches. Technological innovation can be a process to encourage social, cultural, and political innovation. With woman-centered design, we argue that the technology can create a space for different forms of innovation, for real change to be delivered and in support of lines of inquiry that are 'forbidden'. These include the risk of essentialisation: To better understand ourselves we need to acknowledge and design with the biological and engage adequately with our biology [20]. As established before (second-wave feminism) and through current day (third and fourth wave feminism), the body matters. While the biological body might continue to be a part of the great unmentionable, as the "the ghost of biology still haunts us: biological sex, the biological body, remain problematic concepts" (ibid), the biology of the body is also "crucial - and a vital ally - in terms of how we understand both embodiment (our own and others) and the subtly sociopolitical dimensions of scientific knowledge production" [8]. While the female body remains a contested domain, it also persists in association with taboo, made tangible and involved in restrictions that continue to hinder the 
development of women's health and care at their utmost levels of intimacy, e.g. across geographies [100][110] and devices [84]. Our bodily functions and the way our bodies manage themselves might be taken for granted [20]. The association of the body with gross, unthinking physicality [77] through the social significance of natural bodily processes [40] is entangled in qualities of intimate care. These are paramount to health and wellbeing more generally. We have outlined qualities of intimate care elsewhere [5], by situating this care work on the body and across the lifecourse, one which comes into focus when the body's biography is disrupted, and contributes to the construction of the self through bodywork. The body is an ecosystem, and biology is also what includes bodily functions. While some might argue that there are no 'women's bodies' [73], new approaches that look at the body as a living organism explore how processes involved in the materiality of the body, e.g. in its anatomy and physiology, can actively matter and contribute to social and cultural constructs (ibid). The body is a point of departure, and our work has drawn from feminist biology to assimilate notions of embodiment and bodily functions in ways that are conducive to producing knowledge. As in the case of the cis-centric study with Labella, the emphasis was the body materialism through the textile surface and mobile technology, to highlight the dynamic nature of engaging with bodily care. While some have seen our approach as essentialist, we wanted to draw attention to care of the intimate body by exploring biological embodiment (in its processes and the visceral) and by understanding that body in its social context. While doing so, we produced a woman, one that continues to be contested and is shaped by a reality that fits to the mainstream. Whereas women's multiple bodies and identities as women are missing in Labella, we see this reality far from biologically deterministic, as we engage with the body not as a fixed identity nor understand it as merely the result of biological processes.

In our work, we attend to the body as a living organism, one that is being actively remade e.g. by new regimes of pharmaceutics and biotechnologies [97]; the body in its fluidity, one that accounts for matter (e.g. organs) and lived experiences that demand body work e.g. those that require managing the leaky body such as within continence care or breastfeeding. If in doubt of such qualities, one can attest to the dissolution of boundaries between the body and technology - implantable [47], genetic, biologically enhanced, or contraceptive - and how these further challenge perspectives of the body as fixed or unyielding. It is within this flux, as we incorporate self-diagnostic devices and medicating technologies, that new frontiers to the body need to be rescripted. An example would be that in relation to digital technologies. It is well known that technology for (self)tracking the body include those that observe, e.g. sleep patterns, monitor heart rate and regulate exercise, or encourage people to self-track their sexual or reproductive practices. The latter involves intimate care issues targeting mostly 'women's health', e.g. menstruation and fertility, and range from simply offering information or tracking, e.g. ovulation, through capturing personal health and medical data. What these technologies have in common is that on the one hand, they offer a quantitative understanding of one's body and functions, delivered via scientific measurement and interpretation that is much based on biological sex. On the other hand, what they withstand is the discrete factors that include a person's unique experience throughout the course of their lives, including a myriad of personal and unique subjective experiences [62], which include gender and social factors implicit therein. In FeedFinder we offer an illustration of how design can re-frame the body, and in this case the intimate care act of breastfeeding. As a design, rather than the predominant tendency to track and quantify (number of feeds, time spent feeding, which breast was fed from), FeedFinder instead privileges and documents the lived experience of public breastfeeding. The woman produced through FeedFinder is one whose choices are constrained and influenced by societal and cultural factors, recognizing and reflecting back that one's experience of breastfeeding is influenced by more than individual choice and ability. As suggested by Londa Schiebinger in her 2017 talk at the Berlin Institute of Health [19], "sex influences health by modifying behavior and gender behaviors interact with and influence biology". As she claims, "gender behaviors can modify biological factors, for example exercise strengthens bones, and social discrimination can lead to stress, which in turn impacts health (...) these are social factors that differ by gender". There is a myriad of variables that can impact bodies throughout the lifecourse. The woman produced through society is more or less able to breastfeed dependent on how socially, culturally and 
politically permissible such action is. Her body is therefore more of less likely to develop breast and ovarian cancer or type 2 diabetes [114]. FeedFinder produces a woman then who is curious about and willing to describe how breastfeeding is received in her own locale. A woman who is able to potentially challenge the overwhelming message in the British Media that breastfeeding is unwelcome by the British public.

As we reimagine women's health research within HCI we see many potential exciting research which address even more directly the materiality of the human body. We wish to see more uptake and development of interactions which interrelate with the complexity of bio(techno)logy - the human microbiota, which highly impacts human development, physiology, immunity, and nutrition, may be an ideal starting point for such work. More specifically, conditions that are generally associated with the female body, such as menstruation and menopause, are interdependent of hormones such as estrogen and progesterone which in turn contribute to the health of the reproductive system. Hormones are chemical communicators that carry messages to and from different organs in the body, helping the body to remain balanced and function optimally, e.g. hormonal changes have an influence on the vaginal microbiota which can impact fertility or gynecological cancer [25][64]. The role of the vaginal microbiota is indeed increasingly apparent as research shows that it is linked to a number of medical conditions, from bacterial vaginosis to urinary tract infections to sexually transmitted diseases such as HIV [118]. As HCI research has looked at quantified approaches to the body in e.g. menstruation, what is it that the field can do next to open up this space that attends to such intimate material interactions within the body? Can HCI help to quantify fertility within digital technology by incorporating the influence of e.g. the environment or food? We understand the body as an ecosystem and thus a living material, one that is permeable, temporal, situated, vulnerable, one through which we live in and engage with throughout the life course. As Judith Butler advances elsewhere, "we seek to understand ourselves as living creatures bound to human and nonhuman creatures, to entire systems and networks of life" [124]. It is this profound engagement with the world and fragility of boundaries that open up new possibilities for caring and therefore to 'knowing more' [43][78]. To better understand ourselves, we suggest a woman-centered program of research to design that improves the conditions of women, within the social endeavour of (self-) care and through reimagining practices that are paramount throughout the lifecourse and vital to all being in the world. In taking a humanistic approach, we stand by woman as a subject not unified but rather multiple, and accommodating their variety of bodies and identities is critical to advance an agenda in health and care.

\section{To advance equity through design}

By looking at constraints and advancements in women, the body, and health throughout times, we find literature ${ }^{5}$ showing how women's interventions on the human body included exercising midwifery, and being a carer and a carier of traditional knowledge, pivotal to e.g. childbirth experience [87]. It was at a time when such traditional knowledge was challenged by advancements in science and politics that the development of a new image of women as essentially nonscientific was brought to the forefront, and the site of care shifted from the home to the hospital (ibid). In other words, it is with the medicalization of women's health and care that knowledge once belonging to women was discouraged. One example is the case of contraception and that of midwives, who had held the monopoly on the entire field of what would later on come to be designated as women's health. Midwives were removed from practice, e.g. birthing, once the practice became medicalized and was taken over by male physicians with university training. It is within Western countries that this 'advancement', women's loss of control over fertility, has been registered. Furthermore, conceptions that later will be culturally deployed on all women (intertwined with a notion of style) would pave the way to the gendering of e.g. products and services that asserted what woman "always already" was [18]. Engendering women involved an implicit bias and, moving forward in time, becoming engendered as women persists and manifests in terms such as 'the female body' or 'femininity' - experiences in women to traverse

\footnotetext{
${ }^{5}$ We continue by presenting evidence formulated from a Western perspective and as advanced by literature that has focused their analysis on the Global North.
} 
those "underpinned by a core of identification, purity, and mothering - i.e., by the concept of the female body" [81]. While this positioning might have inferred a certain ideal that was fixed in a deeper sociocultural structure, the question of women's bodies, as much as the very category of woman, continue to evolve. We position (health) care in the context of the latter, woman as an evolving categorization and the body as site in flux (that may include the biological or other implicit cultural aspects) to again highlight not only the ecosystem that makes for biology but also that of culture. Attention to gender, equity and human rights can advance health [121]. To advance equity through design we have explored how design and technologies can invite women in generating data, sharing of information, considerations that ease hardship in health and wellbeing (theirs and others). We have previously mentioned data activism, design activism, and health activism in HCI, including e.g. [20][104]. Our approaches have attempted to enable woman to collect their own data and interrogate data that pertain to their own concerns, to provide avenues through which a woman has a voice and say in how a body is understood and cared for by the medical profession, and society and culture more widely, and finally provided long-term research products which benefit the women and communities we have worked with.

We draw from engagements within feminist STS and technoscience to highlight care and how care is intertwined with(in) the body and culture at large. "Care is both necessary to the fabric of biological and social existence and notorious for the problems that it raises when it is defined, legislated, measured, and evaluated. What care looks and feels like is both context-specific and perspective-dependent" [66]. As mentioned in Martin et al., care is a knowledge-making practice, it is active: a practice, an enactment, a doing (ibid). In Lebanon when developing research around food security with Refugee women we see this care as knowledge making practice in action. The women pressed for an accessible outcome of their involvement in the research study. And, working separately and together with the researcher to provide care and support for new refugees entering the Lebanese context through the production of a resource booklet to help new refugees orientate to their new situation. In FeedFinder, we would argue that the act of care for the community is embedded in the act of leaving a review. Leaving a review within the application has no particular value for the person leaving this review, but instead is viewed by FeedFinder users as a way of giving back to the community and supporting breastfeeding women. In Labella, we see care as enabling. In encouraging (and establishing) a well-informed dialogue with her own body, Labella invites the woman to become a participant in the culture and the science of her body, and then to inspire a much-needed active role in the mundane work of performing self-care. To the same extent, couples within Labella also showed how engaging with such a system can be instrumental across sexes and genders. In other words, this latter study calls to learn with, rather than learn about. Sharing bodily knowledge and practices supported them in learning together and be less withdrawn from the other, in ways that could promote their sexual health specifically and health care more generally.

Care is both ontologically and politically ambivalent. It is not a notion to embrace innocently, and both concepts and the labor of care risk essentializing women's experiences. Just as significant, policy and laws that make care-related technologies more or less accessible, e.g. for the pill, and institutional dominance that regulates and determines changes or adequacy in services and e.g. medical devices, can contribute deeply to gender justice and women's rights. Certainly, advances in medical technology occur and so does an increased medicalisation of women's health - medicalised by the pharmaceutical industry and politics [27][42][117]. De-medicalising health [125] - as we attempted through Labella - may render the woman more autonomous over her own body and life more generally [60], providing a woman with knowledge that can lead to lifelong care of the pelvic floor, and the possibility to avoid the "routine" medical interventions and organ removal. As agents of social influence within the relationship doctor and patient, and as gatekeepers to medical knowledge, medical practitioners are key in this process of social change. In research improving access to antenatal care for Syrian refugees in Lebanon, we were able to show how simple interventions that prolong and deepened interactions between women and medical practitioners seemed to also change both the medical 
practitioners and women's perceptions of one another, resulting in more trust and empathy for one another's position [52].

To advance equity through design, we suggest that doing woman-centered design is noteworthy within a field that designs technologies to support, enhance, and improve all human lives. In arguing for a woman-centered approach, we want to emphasize that care, one which is intertwined with policies, politics and labor, needs to attend to the contextual (re)definitions of women's health. The body attended herein is multiple and transformable, and it implicates health care in all women, beyond the binary or binary oppositions. All the same, this approach engages in learning about the other, in which all sexes and genders are to be understood to have the same rights, opportunities, and expectations regarding to both experience and give care. It will be in designing technologies that facilitate cross-disciplinary dialogue in multiple settings and peoples; exploring and developing novel interactions and artefacts that account for disparate communities of women, that will drive social innovation and transform perspectives on societal challenges.

\section{To mitigate disparities}

We recognize that women are not a homogeneous group and that they have different needs and desires, that they find themselves in a wide range of and varied circumstances. What is a Woman, we ask, in order to understand women's multiple identities as women. UN Women [127] works toward gender equality and women's empowerment rather than a redefinition, and offers an answer to our question by advancing that "a woman is anyone who identifies as a woman and is inclusive of cisgender, transgender, gender nonconforming and non-binary people" [128]. In "Am I that Name?", poet and philosopher Denise Riley argued that women are "an old enough project" [80], one that pervades our socio-cultural lived experiences and political meaning-making. Whereas from a philosophical standpoint the 'woman' we have available might be severely damaged (ibid), we also see the dilemma of woman serving to widen the spectrum of opportunities to create new kinds of discourse, new forms of knowledge, and new modes of practice. We want to move beyond socialized gender stereotypes to productively challenge stagnant definitions, and to open avenues for discussion about the design of technologies that account for, e.g techno-social systems or biomedical apparati, which, once revolutionized, can contribute more effectively to improve the quality of life in all and every woman. To mitigate disparities we continue to inquire 'woman' and the agency they have - or do not have - when attending to their health and care more generally. What are the knowledge practices and technologies available to them? As acknowledged earlier, there continue to be challenges to designing technologies for this spectrum of the society - i.e. over half of the world's population, as the World Bank estimates that the number of females worldwide constitutes circa half of the global population [106]; that transgender men's health is a human 'women's' health issue [45], and that health for transgender individuals more broadly includes and is similarly included under women's health [106]. As "health programs are increasingly recognizing that one-size-fits-all does not deliver benefits equitably to all population groups and that in some cases inequity may even be exacerbated" [121], approaches based on gender sensitivity and/or subpopulation, accounting for individual needs and identities, should be considered when designing (with emerging) technologies that can potentially contribute to lessen health inequality and promote wellbeing throughout the lifecourse. To mitigate disparities we have explored design methods that en-gender conversations in health and wellbeing. For example, with Labella we encourage self-knowledge, by engaging the woman in a conversation with her body which intends to produce a better awareness and understanding of her intimate body. This knowledge is delivered through casual and non-specialized digital interactions and an everyday item, a pair of underwear, to enable self-care. We view this approach as quite detached from the prevalent approach to technologies for women's health, which are part of the medical apparatus or, as of late, within the most personal promise of female technologies. And, while personal female health technologies promise to put control in the hands of the 'user' the understandings of the body and its routines is once again hidden this time behind an algorithm. 
If the bodies through which we produce knowledge matter [43][44], and how we know what we know is conditioned by systems of inclusion and exclusion from the communities that configure knowledge [87], it is also the silences and violence implied in the evidences of naming, pinning down, and classifying, that we should pay attention to. Our initial investigations within the realm of digital women's health have been informed by the communities with which we have worked, by the socio-cultural contexts in which they are based, and our own experiences. It is fair to say we have designed from what we know. What we reflect on is a body of work that we have developed with communities of women representative of, e.g., disparate ethnic identities or social class backgrounds, and whose gender reflect that assigned at birth. For the most part, the technologies we proposed responded to a set of culturally ingrained gendered interactions, and involved participants in these studies to use and speculate possible solutions that would be beneficial for them within the social contexts they live in. Technology is not gender neutral, and if in this existing work we did not engage with the complexity of gender, e.g. to include transgender wo/men and non-binary individuals assigned female sex at birth, it is not because we believe these individuals do not have a place within this research agenda. We acknowledge that transgender wo/men and non-binary individuals and groups are routinely marginalized in society and health care [49]. As underserved patient populations, and that these bodies have so far been silenced within our research. We consider it crucial that appropriate methods are developed to discuss such sensitive health topics [79], and that technologies and interactions are designed which respond to the concerns of these communities and individuals. It is within medical literature that we see how little research exists e.g. regarding transgender men's gynecologic care or reproductive needs. This is a group that faces disempowerment via stigma, discrimination, and bias, as well as experiencing numerous health disparities [49]. We see this as research deserving of focus on designing systems and technologies that better attend to these unmet needs.

So far, our body of research aimed first and foremost to highlight existing discrepancies in health care that perpetuate shame and stigma amongst any self-identified ciswoman that normally would have to attend to their bodies throughout the lifecourse or intimate health harrowing events in life: our work strived to support these diverse groups in taking charge and action when it comes to (self-)care and/or the production of knowledge that accounts for their lived bodies. Nevertheless, our research has contributed to opening the space for others, and we consider the concepts that we have developed throughout our research may be extendable to anybody. For example, through Labella we advance the idea of awkward learning, an approach to the design of interactions for coming to know taboo and stigmatized parts of the body. But, we do not know how the approach we took to awkward learning - that of humour and making strange - may operate within conditions such as gender dysmorphia. This is something which should be understood through future research.

To mitigate disparities, we believe woman-centered design should accept and design for the idea of woman as individual and ungeneralizable. We aim as we move forward for a program of research which engages with all kinds of woman, which innovates on designs and technologies for all kinds of woman, recognizing where relevant innovations can be transferred with care to new contexts and concerns.

\section{Conclusion}

Historically, at a time of racial evaluation and matters of physical beauty, beards (or, rather, lack thereof) contributed to place women in nature. Further, this also established that women were objects of knowledge and not necessarily authorized knowers, and as a group of people they were ignored in the production of knowledge even if directly related to them. An ideology of gender assigned women to a "woman's place" and indeed, once universalized woman as the difference from man led to questioning where to fit women, where does she stand in the great chain of being [87]. While this quest(ion) has seen different attempts at being answered throughout history, in this paper we reflect on meanings and knowledge-making of women, 
their bodies and place in contemporarity as we seek to move forward. Situated practices on the body through and with technology may enable or empower women in distinct ways, and this intersects with a multitude of circumstances. In enquiring novel and creative approaches for design that support and enable care in women, we propose woman-centered design as an approach to explore i) the potential of novel and creative ways for $\mathrm{HCI}$ design and research to improve women's experiences in bodily transactions, choices, and rights, and ii) the wider applicability of a woman-centered design methodological approach in, e.g. health, education, public policy, and, importantly, in practices of everyday life. Woman-centered design draws on approaches that implicate women, their bodies and experiences, and technology design that may include e.g. a wide range of digital systems through materials and biotech experiments. As a relatively new field, and one that draws from a multitude of disciplines and that encompasses theory or active cross-collaboration, we see HCI as wellpositioned to push the boundaries of what women's health can be understood as in the future. The body is multiple, and healthcare systems and tools need to account for that. Nonetheless, the large population that self-identifies as women continue to require and demand attention. As we worked within the paradigm of 'woman', to both en-gender and de-gender the scientific body, linguistic choices, the binary, or practices of care rooted in the everyday or the clinic, we aimed to put forward a theoretical and methodological set of procedures that intend to go beyond empathy and traverse areas of the humanistic, activist, and the inclusive. This, if taken into consideration when designing within the space, will provide for better understandings, advance equity, and mitigate disparities not only in women, but also in all peoples.

\section{REFERENCES}

[1] Alex A Ahmed. 2017. Trans Competent Interaction Design: A Qualitative Study on Voice, Identity, and Technology. Interact. Comput. 30, 1 (2017), 53-72. DOI:https://doi.org/10.1093/iwc/iwx018

[2] Teresa Almeida. 2017. Designing Technologies for Intimate Care in Women. Newcastle University, United Kingdom. Retrieved from http://hdl.handle.net/10443/3868

[3] Teresa Almeida. 2019. New Materiality in Intimate Care. In Textile Intersections 2019, 10-17. DOI:https://doi.org/10.17028/rd.lboro.9724634.v1

[4] Teresa Almeida, Ko-Le Chen, Rob Comber, and Madeline Balaam. 2019. Dismantling Feminist Biology through the Design of eTextiles. In RTD2019: Proceedings of the 4th Biennial Research Through Design Conference, 1-15. DOI:https://doi.org/10.6084/m9.figshare.7855805.v1

[5] Teresa Almeida, Rob Comber, and Madeline Balaam. 2016. HCI and Intimate Care as an Agenda for Change in Women's Health. In CHI'16: Proceedings of the 2016 ACM Conference on Human Factors in Computing Systems, 2599-2611. DOI:https://doi.org/10.1145/2858036.2858187

[6] Teresa Almeida, Rob Comber, Gavin Wood, Dean Saraf, and Madeline Balaam. 2016. On Looking at the Vagina through Labella. In CHI'16: Proceedings of the 2016 ACM Conference on Human Factors in Computing Systems, 1810-1821. DOI:https://doi.org/10.1145/2858036.2858119

[7] Teresa Almeida, Marie Louise Juul Søndergaard, Sarah Homewood, Kellie Morrissey, and Madeline Balaam. 2018. Woman-Centred Design. In Book of DRS 2018 Conversations: Catalyst, Proceedings of the DRS2018 Design Research Society International Conference, Sharon Prendeville, Abigail Durrant, Nora O’ Murchú and Keelin Leahy (eds.). Design Research Society, Loughborough University, London, 16-23.

[8] Cecilia Åsberg and Lynda Birke. 2010. Biology is a feminist issue: Interview with Lynda Birke. Eur. J. Women's Stud. 17, 4 (2010), 413-423. DOI:https://doi.org/10.1177/1350506810377696

[9] Cecilia Åsberg and Nina Lykke. 2010. Feminist technoscience studies. Eur. J. Women's Stud. 17, 4 (2010), 299-305. DOI:https://doi.org/10.1177/1350506810377692

[10] Madeline Balaam, Rob Comber, Ed Jenkins, Selina Sutton, and Andrew Garbett. 2015. FeedFinder: A Location-Mapping Mobile Application for Breastfeeding Women. In CHI'15: Proceedings of the 2015 ACM Conference on Human Factors in Computing Systems, 1709-1718. DOI:https://doi.org/10.1145/2702123.2702328

[11] Madeline Balaam, Lone Koe Hansen, Catherine D'Ignazio, Emma Simpson, Teresa Almeida, Stacey Kuznetsov, Mike Catt, and Marie Louise Juul Søndergaard. 2017. Hacking women's health. In CHI'17: EA Proceedings of the 2017 ACM Conference on Human Factors in Computing 
Systems. DOI:https://doi.org/10.1145/3027063.3027085

[12] Madeline Balaam and Lone Koefoed Hansen. 2017. Women's health @ CHI. Interactions 25, 6-7. DOI:https://doi.org/10.1145/3169797

[13] Liam Bannon. 2011. Reimagining HCI: Toward a More Human-Centered Perspective. Interactions, $50-57$.

[14] Jeffrey Bardzell and Shaowen Bardzell. 2015. Humanistic HCI. Synth. Lect. Human-Centered Informatics 8, 4 (2015), 1-185. DOI:https://doi.org/10.2200/S00664ED1V01Y201508HCI031

[15] Jeffrey Bardzell, Shaowen Bardzell, Amanda Lazar, and Norman Makoto Su. 2019. (Re-)Framing Menopause Experiences for HCI and Design. In Proceedings of the 2019 CHI Conference on Human Factors in Computing Systems (CHI '19), Paper 115.

DOI:https://doi.org/10.1145/3290605.3300345

[16] Jeffrey Bardzell, Shaowen Bardzell, Carl Disalvo, Bill Gaver, and Phoebe Sengers. 2012. Panel: The Humanities and / in HCI. In Proc. of the 2012 CHI Conference on Human Factors in Computing Systems - CHI'12 Extended Abstracts, 1135-1138.

[17] Shaowen Bardzell. 2010. Feminist HCI: Taking Stock and Outlining an Agenda for Design. In Proceedings of the 2010 CHI Conference on Human Factors in Computing Systems - CHI '10, 1301-1310.

[18] Lauren Berlant. 1998. The Female Complaint. Soc. Text 19 (1998), 237-259.

[19] Berlin Institute of Health. 2017. Gendered Innovations in Health and Medicine. Retrieved from https://www.youtube.com/watch?v=x95Rj9q6qS8

[20] Lynda Birke. 1999. Feminism and the Biological Body (Gender, Sc ed.). Edinburgh University Press, Edinburgh.

[21] Chris Bobel. 2010. New Blood: Third-Wave Feminism and the Politics of Menstruation. Rutgers University Press, Piscataway, NJ.

[22] Judith Butler. 2011. Speaking Up, Talking Back. In The Question of Gender: Joan W. Scott's Critical Feminism, Judith Butler and Elizabeth Weed (eds.). Indiana University Press, Bloomington and Indianapolis, 11-27.

[23] Sheelagh Carpendale, Shaowen Bardzell, Margaret Burnett, Neha Kumar, and Madeline Balaam. 2018. Panel: Extending Conversations about Gender and HCI. In EA Proceedings of the SIGCHI Conference on Human Factors in Computing Systems, 1-6.

DOI:https://doi.org/10.1145/3170427.3186325

[24] Justine Cassell. 2002. Genderizing HCI. In The Handbook of Human-Computer Interaction. Lawrence Erlbaum, Mahwah, NJ, 402-411.

[25] M Champer, A M Wong, J Champer, I L Brito, P W Messer, J Y Hou, and J D Wright. 2017. The role of the vaginal microbiome in gynaecological cancer. Obstet. Gynaecol. 125, (2017), 309-315. DOI:https://doi.org/10.1111/1471-0528.14631

[26] Shauna Julia Concannon, Madeline Balaam, Emma Simpson, and Rob Comber. 2018. Applying Computational Analysis to Textual Data from the Wild: A Feminist Perspective. In Proc. of the 2018 CHI Conference on Human Factors in Computing Systems - CHI'18, 1-13.

[27] Peter Conrad. 2013. Medicalization: Changing Contours, Characteristics, and Contexts. In Medical Sociology on the Move, W. C. Cockerham (ed.). Springer, Dordrecht.

DOI:https://doi.org/10.1007/978-94-007-6193-3

[28] Caroline Criado Perez. 2019. Invisible Women : Exposing Data Bias in a World Designed for Men. Vintage Publishing, London.

[29] Hannah Devlin. 2017. Vaginal mesh implants: "If I lift my leg my whole body shakes." The Guardian. Retrieved October 20, 2019 from https://www.theguardian.com/society/2017/oct/19/vaginal-mesh-implants-if-i-lift-my-leg-mywhole-body-shakes

[30] Hannah Devlin. 2017. Vaginal mesh implants: "I really thought I was dying." The Guardian. Retrieved October 20, 2019 from https://www.theguardian.com/society/2017/aug/15/vaginal-meshimplants-i-really-thought-i-was-dying

[31] Hannah Devlin. 2017. Revealed: Johnson \& Johnson's "irresponsible" actions over vaginal mesh implant. The Guardian. Retrieved October 20, 2019 from https://www.theguardian.com/society/2017/sep/29/revealed-johnson-johnsons-irresponsibleactions-over-vaginal-mesh-implant

[32] Hannah Devlin. 2018. Vaginal mesh review to investigate true scale of complications. The 
Guardian. Retrieved October 20, 2019 from

https://www.theguardian.com/society/2018/jan/30/vaginal-mesh-review-to-investigate-true-scaleof-complications

[33] Markéta Dolejšová and Kaiying Lin. 2015. StreetSauce: Taste Interaction and Empathy with Homeless People. In Proc. of the 2015 CHI Conference on Human Factors in Computing Systems Extended Abstracts, 1247-1252.

[34] Sheena Erete, Aarti Israni, and Tawanna Dillahunt. 2017. An Intersectional Approach to Designing in the Margins. Interactions, 66-69.

[35] Sarah Fox, Amanda Menking, Stephanie Steinhardt, Anna Lauren Hoffman, and Shaowen Bardzell. 2017. Imagining Intersectional Futures: Feminist approaches in CSCW. In Proc CSCW '17 Companion, 387-393.

[36] Sarah Fox, Rachel Rose Ulgado, and Daniela K Rosner. 2015. Hacking Culture, Not Devices: Access and Recognition in Feminist Hackerspaces. In CSCW 2015, 56-68.

[37] Catherine Gallagher and Thomas Laqueur. 1987. The Making of the Modern Body Sexuality and Society in the Nineteenth Century. University of California Press.

[38] Moira Gatens. 2003. Imaginary Bodies: Ethics, power, and corporeality. Taylor \& Francis eLibrary. DOI:https://doi.org/10.4324/9780203418659

[39] Stacy Gillis and Rebecca Munford. 2003. Harvesting our strengths: Third wave feminism and women's studies. J. Int. Womens. Stud. 4, 2 (2003), 1-6. Retrieved from http://www.scopus.com/inward/record.url?eid=2-s2.0-18444405195\&partnerID=tZOtx3y1

[40] Elizabeth Grosz. 1994. Sexed Bodies. In Volatile Bodies: Towards a Corporeal Feminism (Theories o). Indiana University Press.

[41] Oliver L Haimson. 2018. Social Media as Social Transition Machinery. In Proceedings of the ACM on Human-Computer Interaction, CSCW, Article 63.

[42] Drew Halfmann. 2011. Recognizing medicalization and demedicalization: Discourses, practices, and identities. Health (Irvine. Calif). May (2011). DOI:https://doi.org/10.1177/1363459311403947

[43] Donna Haraway. 1988. Situated Knowledges: The Science Question in Feminism and the Privilege of Partial Perspective. Fem. Stud. 14, 3 (1988), 575. DOI:https://doi.org/10.2307/3178066

[44] Sandra Harding. 1991. Whose Science? Whose Knowledge? Cornell University Press, Ithaca.

[45] E. Cameron Hartofelis and Anu Manchikanti Gomez. 2013. Trans Men's Health is a "Women's Health" Issue: Expanding the Boundaries of Sexual \& Reproductive Health Care. National Women's Health Network. Retrieved May 13, 2019 from https://www.nwhn.org/trans-mens-healthis-a-womens-health-issue-expanding-the-boundaries-of-sexual-reproductive-health-care/

[46] Heart \& Stroke Foundation of Canada. 2018. Ms.Understood: Women's hearts are victims of a system that is ill-equiped to diagnose, treat and support them. Retrieved from https://www.heartandstroke.ca/-/media/pdf-files/canada/2018-heart-month/hs_2018-heartreport_en.ashx?la=en\&hash=3BBC7F1DD1DA3EDC6B2B0E9BB31E855268C051EB

[47] Kayla J Heffernan, Frank Vetere, and Shanton Chang. 2016. You Put What, Where? Hobbyist Use of Insertable Devices. In Proc. of the 2016 CHI Conference on Human Factors in Computing Systems, 1798-1809.

[48] Clare Hemmings. 2012. Affective solidarity: Feminist reflexivity and political transformation. Fem. Theory 13, 2 (2012), 147-161. DOI:https://doi.org/10.1177/1464700112442643

[49] Alexis Hoffkling, Juno Obedin-maliver, and Jae Sevelius. 2017. From erasure to opportunity: a qualitative study of the experiences of transgender men around pregnancy and recommendations for providers. BMC Pregnancy Childbirth 17, Suppl 2 (2017), 7-20.

DOI:https://doi.org/10.1186/s12884-017-1491-5

[50] Sarah Homewood. 2018. Reframing Design Problems Within Women's Health. In Proceedings of DRS2018 Design Research Society International Conference.

DOI:https://doi.org/10.21606/dma.2017.337

[51] Sarah Homewood. 2019. Inaction as a Design Decision : Reflections on Not Designing SelfTracking Tools for Menopause. In Proc. of the 2019 CHI Conference on Human Factors in Computing Systems - CHI'19 Extended Abstracts, 1-12.

[52] Sarah Homewood, Harvey Bewley, and Laurens Boer. 2019. Ovum : Designing for Fertility Tracking as a Shared and Domestic Experience. In Proceedings of the Conference on Designing Interactive Systems (DIS '19).

[53] Deborah G. Johnson. 2010. Sorting Out the Question of Feminist Technology. In Feminist 
Technology (Women, Gen), Linda L. Layne, Sharra L. Vostral and Kate Boyer (eds.). University of Illinois Press, 36-54.

[54] Stacey Kuznetsov, Alex S. Taylor, Tim Regan, Nicolas Villar, and Eric Paulos. 2012. At the seams: DIYbio and opportunities for HCI. In Proceedings of the Conference on Designing Interactive Systems (DIS '12), 258-267.

[55] Cristine Labuski. 2008. Virginal Thresholds. In Luce Irigaray: Teaching, Luce Irigaray and Mary Green (eds.). Continuum, New York, NY, 13-23.

[56] Marie Larsson. 2019. INFERIOR: how science got women wrong — and the new research that's rewriting the story. NORA - Nord. J. Fem. Gend. Res. 27, 1 (2019), 73-76.

DOI:https://doi.org/10.1080/08038740.2019.1576371

[57] Teresa De Lauretis. 1987. The Technology of Gender. In Technologies of Gender. Indiana University Press, Bloomington, $1-30$.

[58] Amanda Lazar, Norman Makoto Su, Jeffrey Bardzell, and Shaowen Bardzell. 2019. Parting the Red Sea: Sociotechnical Systems and Lived Experiences of Menopause. In Proceedings of the 2019 CHI Conference on Human Factors in Computing Systems - CHI '19, Paper No. 480. DOI:https://doi.org/10.1145/3290605.3300710

[59] Sara Ljungblad and Lars Erik Holmquist. 2007. Transfer Scenarios: Grounding Innovation with Marginal Practices. In Proceedings of the 2007 CHI Conference on Human Factors in Computing Systems - CHI '07.

[60] Paul A Lombardo. 2015. How to Escape the Doctor's Dilemma? De-Medicalize Reproductive Technologies. J. Law, Med. Ethics Summer (2015), 326-329.

[61] Donna Lu. 2019. The femtech gold rush. New Scientist, 20-21.

[62] Deborah Lupton. 2014. Quantified sex: a critical analysis of sexual and reproductive self-tracking using apps. Cult. Heal. Sex. An Int. J. Res. , Interv. Care February 2015 (2014), 37-41. DOI:https://doi.org/10.1080/13691058.2014.920528

[63] Celia Lury and Nina Wakeford (Eds.). 2014. Inventive Methods: The happening of the social. Routledge.

[64] Bing Ma, Larry J. Forney, and Jacques Ravel. 2012. The vaginal microbiome: rethinking health and diseases. Annu. Rev. Microbiol. 66, (2012), 371-389.

[65] Fiona MacGregor. 2015. Myanmar: women's fight against verbal taboo symbolises wider rights battle. The Guardian. Retrieved June 12, 2019 from https://www.theguardian.com/globaldevelopment/2015/nov/02/myanmar-womens-fight-against-verbal-taboo-symbolises-wider-rightsbattle

[66] Aryn Martin, Natasha Myers, and Ana Viseu. 2015. The politics of care in technoscience. Soc. Stud. Sci. 45, 5 (2015), 625-641. DOI:https://doi.org/10.1177/0306312715602073

[67] Ramia Mazé, Laura Forlano, Li Jonsson, Kristina Lindström, and Åsa Ståhl. 2018. Design, Research and Feminism(s). DRS2018. Retrieved May 6, 2019 from http://www.drs2018limerick.org/track/design-research-and-feminisms

[68] Merriam Webster. 2018. Woman. Merriam Webster. Retrieved from https://www.merriamwebster.com/dictionary/woman

[69] Lydia Michie, Madeline Balaam, John Mccarthy, Timur Osadchiy, and Kellie Morrissey. 2018. From Her Story, to Our Story: Digital Storytelling as Public Engagement around Abortion Rights Advocacy in Ireland. In Proceedings of the 2018 CHI Conference on Human Factors in Computing Systems - CHI '18, 1-15.

[70] Shari Munch. 2004. Gender-Biased Diagnosing of Women's Medical Complaints: Contributions of Feminist Thought, 1970 - 1995. Women Health 40, 1 (2004), 101-121. DOI:https://doi.org/10.1300/J013v40n01

[71] Shari Munch. 2006. The women's health movement: making policy, 1970-1995. Soc. Work Health Care 43, 1 (2006), 17-32. DOI:https://doi.org/10.1300/J010v43n01

[72] Michelle Murphy. 2012. Seizing the Means of Reproduction: Entanglements of Feminism, Health, and Technoscience (Experiment ed.). Duke University Press, Durham and London.

[73] Nelly Oudshoorn. 1999. The decline of the one-size-fits-all paradigm, or, how reproductive scientists try to cope with postmodernity. In The social shaping of technology (Second edi), Donald MacKenzie and Judy Wajcman (eds.). Open University Press, UK, 325-340.

[74] Nelly Oudshoorn, Ann Rudinow Saetnan, and Merete Lie. 2002. On Gender and Things: Reflections on an Exhibition on Gendered Artifacts. Women's Stud. Int. Forum 25, 4 (2002), 471- 
483.

[75] Andrea G Parker, Vasudhara Kantroo, Hee Rin Lee, Miguel Osornio, Mansi Sharma, and Rebecca E Grinter. 2012. Health Promotion as Activism: Building Community Capacity to Effect Social Change. In Proc. of the 2012 CHI Conference on Human Factors in Computing Systems - CHI'12, 99-108.

[76] Luiza Prado de O. Martins. 2014. Privilege and Oppression: Towards a Feminist Speculative Design. In Proceedings of DRS 2014: Design Research Society International Conference, 98-990.

[77] Janet Price and Margrit Shildrick (Eds.). 1999. Feminist Theory and the body. Edinburgh University Press.

[78] María Puig de la Bellacasa. 2017. Mtters of Care: Speculative Ethics in More Than Human Worlds (Posthumani ed.). University of Minnesota Press.

[79] Sari L Reisner, Renee K Randazzo, Jaclyn M White Hughto, Sarah Peitzmeier, Zachary Dubois, Dana J Pardee, Elliot Marrow, Sarah Mclean, and Jennifer Potter. 2018. Sensitive Health Topics With Underserved Patient Populations: Methodological Considerations for Online Focus Group Discussions. Qual. Health Res. 28, 10 (2018), 1658-1673. DOI:https://doi.org/10.1177/1049732317705355.Sensitive

[80] Denise Riley. 1993. "Am I That Name?": Feminism and the category of "Women" in History (Language, ed.). The MacMillan Press Ltd., London.

[81] Denise Riley. 1999. Bodies, Identities, Feminisms. In Feminist Theory and the Body: A Reader, Janet Price and Margrit Shildrick (eds.). Edinburgh University Press, 220-226.

[82] Jennifer A Rode. 2011. A theoretical agenda for feminist HCI. Interact. Comput. 23, 5 (2011), 393 400. DOI:https://doi.org/10.1016/j.intcom.2011.04.005

[83] Celia Rosas. 2019. The Future is Femtech: Privacy and Data Security Issues Surrounding Femtech Applications. Hast. Bus. Law J. 15, 2 (2019).

[84] Jennifer Stroud Rossmann. 2008. Built to Spec"? The vaginal speculum as a case study of inadequate design. Ambidextrous, 47-49.

[85] Dana Roth. 2018. Gender Bias in Clinical Drug Trials. Gend. Soc. 83, (2018).

[86] Angela Saini. 2017. INFERIOR: How Science Got Women Wrong-and the New Research That's Rewriting the Story. Beacon Press.

[87] Londa Schiebinger. 1993. Nature's Body: Gender in the Making of Modern Science. Beacon Press, Boston.

[88] Londa Schiebinger. 1999. Theories of Gender and Race. In Feminist Theory and the Body: A Reader, Janet Price and Margrit Shildrick (eds.). Edinburgh University Press, 21-31.

[89] Ari Schlesinger, W. Keith Edwards, and Rebecca E. Grinter. 2017. Intersectional HCI: Engaging Identity through Gender, Race, and Class. In Proceedings of the 2017 CHI Conference on Human Factors in Computing Systems - CHI '17, 5412-5427.

DOI:https://doi.org/10.1145/3025453.3025766

[90] Joan W Scott. 1986. Gender : A Useful Category of Historical Analysis. Am. Hist. Rev. 91, 5 (1986), 1053-1075.

[91] Margrit Shildrick and Janet Price. 1999. Openings on the Body: A Critical Introduction. In Feminist Theory and the Body: A Reader, Janet Price and Margrit Shildrick (eds.). Edinburgh University Press, Edinburgh, 1-14.

[92] Emma Simpson, Rob Comber, Andrew Garbett, Ed Ian Jenkins, and Madeline Balaam. 2017. Experiences of Delivering a Public Health Data Service. In Proceedings of the 2017 ACM annual conference on Human Factors in Computing Systems - CHI '17, 6171-6183.

DOI:https://doi.org/10.1145/3025453.3025881

[93] Emma Simpson, Andrew Garbett, Rob Comber, and Madeline Balaam. 2015. Factors important for women who breastfeed in public: a content analysis of review data from FeedFinder. BMJ Online (2015).

[94] Dale Spender. 1990. Man Made Language (2 edition ed.). Pandora Press.

[95] Katta Spiel, Os Keyes, and Pinar Barlas. 2019. Patching Gender: Non-binary Utopias in HCI. In Proc. of the 2018 CHI Conference on Human Factors in Computing Systems - CHI'18 Extended Abstracts, 1-11.

[96] Denny L Starks, Tawanna Dillahunt, and Oliver L Haimson. 2019. Designing Technology to Support Safety for Transgender Women \& Non-Binary People of Color. In Proceedings of the 2019 on Designing Interactive Systems International Conference (DIS '19 Companion), 289-294. 
[97] Elizabeth Stephens. 2014. Feminism and New Materialism: The Matter of Fluidity. Queer Stud. 9, special issue: bodily fluids (2014), 186-202. Retrieved from http://interalia.org.pl

[98] Angelika Strohmayer, Mary Laing, and Rob Comber. 2017. Technologies and Social Justice Outcomes in Sex Work Charities: Fighting Stigma, Saving Lives. In Proceedings of the 2017 CHI Conference on Human Factors in Computing Systems - CHI '17, 3352-3364.

[99] Laurel Stvan. 2016. That's what zhe said: mx-ing up the language of gende. The Conversation UK. Retrieved May 3, 2019 from https://theconversation.com/thats-what-zhe-said-mx-ing-up-thelanguage-of-gender51963? fbclid=IwAR1eq7iZQ9gFO3yu_AwympGFxBNVx5M2xYsBordrMzG1Lr5js9eoQV6nCT0

[100] Sharifa Sultana, François Guimbretière, Phoebe Sengers, and Nicola Dell. 2018. Design Within a Patriarchal Society: Opportunities and Challenges in Designing for Rural Women in Bangladesh. In Proceedings of the 2018 CHI Conference on Human Factors in Computing Systems (CHI '18), 113. DOI:https://doi.org/10.1145/3173574.3174110

[101] Reem Talhouk, Madeline Balaam, Austin L Toombs, Andrew Garbett, Chaza Akik, Hala Ghattas, Vera Araujo-Soares, Balsam Ahmad, and Kyle Montague. 2019. Involving Syrian Refugees in Design Research: Lessons Learnt from the Field. In Proceedings of the 2019 Conference on Designing Interactive Systems (DIS'19), 1583-1594.

DOI:https://doi.org/10.1145/3322276.3322335

[102] Reem Talhouk, Tom Bartindale, Kyle Montague, S. Mesmar, Chaza Akik, M. Najem, H. Ghattas, A. Ghassani, Patrick Olivier, and Madeline Balaam. 2017. Implications of Synchronous IVR Radio on Syrian Refugee Health and Community Dynamics. In Proceedings of the 8th International Conference on Communities and Technologies (C\&T '17), 193-202. DOI:https://doi.org/10.1145/3083671.3083690

[103] Reem Talhouk, Sandra Mesmar, Anja Thieme, Madeline Balaam, Patrick Olivier, Chaza Akik, and Hala Ghattas. 2016. Syrian Refugees and Digital Health in Lebanon: Opportunities for Improving Antenatal Health. In 2016 CHI Conference on Human Factors in Computing Systems, 331-342. DOI:https://doi.org/10.1109/TIE.2017.2721882

[104] Reem Talhouk, Kellie Morrissey, Sarah Fox, Nadia Pantidi, Emma Simpson, Lydia Emma Michie, and Madeline Balaam. 2018. Human Computer Interaction \& Health Activism. In Proceedings of the 2018 CHI Conference on Human Factors in Computing Systems - CHI Extended Abstracts '18, $1-4$.

[105] V. Tanner. 2016. Why We Must Stop Calling Menstruation a 'Women's Issue.' Medium. Retrieved October 28, 2019 from https://medium.com/the-establishment/why-we-must-stop-callingmenstruation-a-womens-issue-a9c58c677f7c

[106] The American College of Obstetricians and Gynecologists. Health care for transgender individuals. Comm. Heal. Care Underserved Women Committee, DOI:https://doi.org/10.1097/AOG.0b013e31823ed1c1

[107] Giulia Tomasello. Future Flora. Retrieved May 20, 2019 from https://gitomasello.com/Future-Flora

[108] Giulia Tomasello and Teresa Almeida. 2020. Empowerment and Self-Care: Designing for the Female Body. In Crafting Anatomies: Archives, Dialogues, Fabrications, Katherine Townsend, Rhian Solomon and Amanda Briggs-Goode (eds.). Bloomsbury Publishing, London, UK, 171-188.

[109] Nancy Tuana. 2006. The Speculum of Ignorance: The Women's Health Movement and Epistemologies of Ignorance. Hypatia, A J. Fem. Philos. 21, 3 (2006), 1-19. DOI:https://doi.org/https://doi.org/10.1111/j.1527-2001.2006.tb01110.x

[110] Anupriya Tuli, Shaan Chopra, Neha Kumar, and Pushpendra Singh. 2018. Learning from and with Menstrupedia: Towards Menstrual Health Education in India. In Proceedings of the ACM on Human-Computer Interaction, 1-20. DOI:https://doi.org/10.1145/3274443

[111] Agatha Tutia, Lan Vu, Kelda Baljon, and Daniela K Rosner. 2019. HCI and Menopause: Designing With and Around the Aging Body. In Proc. of the 2019 CHI Conference on Human Factors in Computing Systems - CHI'19 Extended Abstracts, 1-8.

[112] UNFPA. 2019. Menstruation and human rights - Frequently asked questions. UNFPA. Retrieved October 28, 2019 from https://www.unfpa.org/menstruationfaq

[113] Stanford University. 2019. Gendered Innovations in Science, Health \& Medicine, Engineering, and Environment. Retrieved May 3, 2019 from https://genderedinnovations.stanford.edu/

[114] Cesar G Victora, Rajiv Bahl, Aluísio J D Barros, Giovanny V A França, Susan Horton, Julia Krasevec, Simon Murch, Mari Jeeva Sankar, Neff Walker, and Nigel C Rollins. 2016. 
Breastfeeding in the 21st century: epidemiology, mechanisms, and lifelong effect. Lancet 387 , 10017 (2016), 475-490.

[115] Judy Wajcman. 2007. From women and technology to gendered technoscience. Information, Community Soc. 10, 3 (2007), 287-298. DOI:https://doi.org/10.1080/13691180701409770

[116] Judy Wajcman. 2010. Feminist theories of technology. Cambridge J. Econ. 34, (2010), 143-152. DOI:https://doi.org/10.1093/cje/ben057

[117] Alistair Wardrope. 2015. Medicalization and epistemic injustice. Med. Heal. Care Philos. (2015), 341-352. DOI:https://doi.org/10.1007/s11019-014-9608-3

[118] Jocelyn M. Wessels, Allison M. Felker, Haley A. Dupont, and Charu Kaushic. 2018. The relationship between sex hormones, the vaginal microbiome and immunity in HIV-1 susceptibility in women. Dis. Model. Mech. 11, 9 (2018).

[119] Pamela J. Wisniewski, Neha Kumar, Christine Bassem, Sarah Clinch, Susan M. Dray, Geraldine Fitzpatrick, Cliff Lampe, Michael Muller, and Anicia N. Peters. 2018. Intersectionality as a Lens to Promote Equity and Inclusivity within SIGCHI. In Proceedings of the 2018 CHI Conference on Human Factors in Computing Systems (CHI '18), panel08. DOI:https://doi.org/10.1145/3170427.3186324

[120] Marisol Wong-Villacres, Arkadeep Kumar, Aditya Vishwanath, Naveena Karusala, Betsy DiSalvo, and Neha Kumar. 2018. Designing for Intersections. In Proceedings of the Conference on Designing Interactive Systems (DIS '18), 45-58.

[121] World Health Organization. 2017. Advancing Health through Attention to Gender, Equity and Human Rights.

[122] Nadia Campo Woytuk, Mingxing Liu, and Linette Nilsson. 2019. Your Period Rules : Design Implications for Period-Positive Technologies. In Proceedings of the 2019 CHI Conference on Human Factors in Computing Systems (CHI '19)- Extended Abstracts.

[123] Peter Wright and John McCarthy. 2008. Empathy and experience in HCI. In Proc. CHI '08, 637. DOI:https://doi.org/10.1145/1357054.1357156

[124] George Yancy. 2019. Judith Butler: When Killing Women Isn't a Crime. The New York Times. Retrieved October 20, 2019 from https://www.nytimes.com/2019/07/10/opinion/judith-butlergender.html?fbclid=IwAR3BE_maD-3T-iKXbFqnaoCHmXyLecgzTSiHvYzlF-oKUPL2lyKP603$\mathrm{xDk}$

[125] Emily Yates-doerr and Megan A Carney. 2015. Demedicalizing Health: The Kitchen as a Site of Care. Med. Anthropol. 9740, October (2015). DOI:https://doi.org/10.1080/01459740.2015.1030402

[126] Alyson L Young and Andrew D Miller. 2019. " This Girl is on Fire " Sensemaking in an Online Health Community for Vulvodynia. In Proceedings of the 2019 CHI Conference on Human Factors in Computing Systems (CHI '19), 1-13.

[127] UN Women. Retrieved May 8, 2019 from http://www.unwomen.org/en

[128] AmplifyChange. Retrieved May 8, 2019 from https://amplifychange.org/ 